\title{
THE CHURCH SYNTHESIS PROBLEM WITH PARAMETERS
}

\author{
ALEXANDER RABINOVICH
}

Sackler Faculty of Exact Sciences, Tel Aviv University, Israel 69978.

e-mail address: rabinoa@post.tau.ac.il

\begin{abstract}
For a two-variable formula $\psi(X, Y)$ of Monadic Logic of Order $(M L O)$ the Church Synthesis Problem concerns the existence and construction of an operator $Y=$ $F(X)$ such that $\psi(X, F(X))$ is universally valid over $N a t$.

Büchi and Landweber proved that the Church synthesis problem is decidable; moreover, they showed that if there is an operator $F$ that solves the Church Synthesis Problem, then it can also be solved by an operator defined by a finite state automaton or equivalently by an $M L O$ formula. We investigate a parameterized version of the Church synthesis problem. In this version $\psi$ might contain as a parameter a unary predicate $P$. We show that the Church synthesis problem for $P$ is computable if and only if the monadic theory of $\langle N a t,\langle, P\rangle$ is decidable. We prove that the Büchi-Landweber theorem can be extended only to ultimately periodic parameters. However, the $M L O$-definability part of the BüchiLandweber theorem holds for the parameterized version of the Church synthesis problem.
\end{abstract}

\section{INTRODUCTION}

Two fundamental results of classical automata theory are decidability of the monadic second-order logic of order $(\mathrm{MLO})$ over $\omega=($ Nat,$<)$ and computability of the Church synthesis problem. These results have provided the underlying mathematical framework for the development of formalisms for the description of interactive systems and their desired properties, the algorithmic verification and the automatic synthesis of correct implementations from logical specifications, and advanced algorithmic techniques that are now embodied in industrial tools for verification and validation.

Büchi [Bu60] proved that the monadic theory of $\omega=\langle N a t,<\rangle$ is decidable. Even before the decidability of the monadic theory of $\omega$ has been proved, it was shown that the expansions of $\omega$ by "interesting" functions have undecidable monadic theory. In particular, the monadic theory of $\langle N a t,<,+\rangle$ and the monadic theory of $\langle N a t,<, \lambda x .2 \times x\rangle$ are undecidable [Rob58, Trak61]. Therefore, most efforts to find decidable expansions of $\omega$ deal with expansions of $\omega$ by monadic predicates.

Elgot and Rabin [ER66] found many interesting predicates $\mathbf{P}$ for which $M L O$ over $\langle$ Nat, $<, \mathbf{P}\rangle$ is decidable. Among these predicates are the set of factorial numbers $\{n !: n \in$ $N a t\}$, the sets of $k$-th powers $\left\{n^{k}: n \in N a t\right\}$ and the sets $\left\{k^{n}: n \in N a t\right\}$ (for $k \in N a t$ ).

1998 ACM Subject Classification: F.4.1;F.4.3.

Key words and phrases: Synthesis Problem, Decidability, Monadic Logic.

드 IN LOGICAL METHODS
DOI:10.2168/LMCS-3 (4:9) 2007
(C) A. Rabinovich (c) Creative Commons 
The Elgot and Rabin method has been generalized and sharpened over the years and their results were extended to a variety of unary predicates (see e.g., [Ch69, Th75, Sem84, CT02]). In [Rab05, Rab07, RT06] we provided necessary and sufficient conditions for the decidability of monadic (second-order) theory of expansions of the linear order of the naturals $\omega$ by unary predicates.

Let Spec be a specification language and $\mathrm{Pr}$ be an implementation language. The synthesis problem for these languages is stated as follows: find whether for a given specification $S(I, O) \in$ SPEC there is a program $\mathcal{P}$ which implements it, i.e., $\forall I(S(I, \mathcal{P}(I))$.

The specification language for the Church Synthesis problem is the Monadic secondorder Logic of Order. An $M L O$ formula $\varphi(X, Y)$ specifies a binary relation on subsets of Nat. Note that every subset $P$ of $N a t$ is associated with its characteristic $\omega$-string $u_{P}$ (where $u_{P}(i)=1$ if $i \in P$ and otherwise $u_{P}(i)=0$ ). Hence, $\varphi(X, Y)$ can be considered as a specification of a binary relation on $\omega$-strings.

As implementations, Church considers functions from the set $\{0,1\}^{\omega}$ of $\omega$-strings over $\{0,1\}$ to $\{0,1\}^{\omega}$. Such functions are called operators. A machine that computes an operator at every moment $t \in N a t$ reads an input symbol $X(t) \in\{0,1\}$ and produces an output symbol $Y(t) \in\{0,1\}$. Hence, the output $Y(t)$ produced at $t$ depends only on inputs symbols $X(0), X(1), \ldots, X(t)$. Such operators are called causal operators (C-operators); if the output $Y(t)$ produced at $t$ depends only on inputs symbols $X(0), X(1), \ldots, X(t-1)$, the corresponding operator is called strongly causal (SC-operator). The sets of recursive causal and strongly causal operators are defined naturally; a $\mathrm{C}$-or a SC-operator is a finite state operator if it is computable by a finite state automaton (for precise definitions, see Subsection 4.4).

The following problem is known as the Church Synthesis problem.

Church Synthesis problem
Input: an $M L O$ formula $\psi(X, Y)$.
Task: Check whether there is a C-operator $F$ such that
Nat $\models \forall X \psi(X, F(X))$ and if so, construct this operator.

The Church Synthesis problem is much more difficult than the decidability problem for $M L O$ over $\omega$. Büchi and Landweber BL69 proved that the Church synthesis problem is computable. Their main theorem is stated as follows:

Theorem 1.1. For every $M L O$ formula $\psi(X, Y)$ either there is a finite state $C$-operator $F$ such that $N a t \models \forall X \psi(X, F(X))$ or there is a finite state $S C$-operator $G$ such that $N a t \models$ $\forall Y \neg \psi(G(Y), Y)$. Moreover, it is decidable which of these cases holds and a corresponding operator is computable from $\psi$.

In this paper we consider natural generalizations of the Church Synthesis Problem over expansions of $\omega$ by monadic predicates, i.e., over the structures $\langle$ Nat, $<, \mathbf{P}\rangle$.

For example, let $\mathbf{F a c}=\{n !: n \in N a t\}$ be the set of factorial numbers, and let $\varphi(X, Y$, Fac $)$ be a formula which specifies that $t \in Y$ iff $t \in$ Fac and $\left(t^{\prime} \in X\right) \leftrightarrow$ $\left(t^{\prime} \in \mathbf{F a c}\right)$ for all $t^{\prime} \leq t$. It is easy to observe that there is no finite state C-operator $F$ such that $\forall X \varphi(X, F(X), \mathbf{F a c})$. However, there is a recursive C-operator $H$ such that $\forall X \varphi(X, H(X), \mathbf{F a c})$. It is also easy to construct a finite state C-operator $G(X, Z)$ such that $\forall X \varphi(X, G(X, \mathbf{F a c}), \mathbf{F a c})$. It was surprising for us to discover that it is decidable whether for a formula $\psi(X, Y, \mathbf{F a c})$ there is a C-operator $F$ such that $\forall X \varphi(X, F(X)$, Fac $)$ and if such an operator exists, then it is recursive and computable from $\psi$. 
Here is the summary of our results. We investigate a parameterized version of the Church synthesis problem. In this version $\psi$ might contain as a parameter a unary predicate $P$. Below five synthesis problems with a parameter $\mathbf{P} \subseteq N a t$ are stated. We use capital italic letters for unary predicate names and set variables and the corresponding bold letters for their interpretation.

Synthesis Problems for $\mathbf{P} \subseteq$ Nat
Input: an $M L O$ formula $\psi(X, Y, P)$.
Problem 1: Check whether there is a C-operator $Y=F(X, P)$ such that
Nat $\models \forall X \psi(X, F(X, \mathbf{P}), \mathbf{P})$ and if there is such a recursive operator
- construct it.
Problem 2: Check whether there is a recursive C-operator $Y=F(X, P)$ such
that $N a t \models \forall X \psi(X, F(X, \mathbf{P}), \mathbf{P})$ and if so - construct this operator.
Problem 3: Check whether there is a recursive C-operator $Y=F(X)$ such
that $N a t \models \forall X \psi(X, F(X), \mathbf{P})$ and if so - construct this operator.

The next two problems are obtained from problems 2 an 3 when "recursive" is replaced by "finite state".

Synthesis Problems for $\mathbf{P} \subseteq$ Nat
Input: an $M L O$ formula $\psi(X, Y, P)$.
Problem 4: Check whether there is a finite state C-operator $Y=F(X, P)$ such
that $N a t \models \forall X \psi(X, F(X, \mathbf{P}), \mathbf{P})$ and if so - construct this operator.
Problem 5: Check whether there is a finite state C-operator $Y=F(X)$ such
that $N a t \models \forall X \psi(X, F(X), \mathbf{P})$ and if so - construct this operator.

We show

Theorem 1.2. Let $\mathbf{P}$ be a subset of Nat. The following conditions are equivalent:

(1) Problem 1 for $\mathbf{P}$ is computable.

(2) Problem 2 for $\mathbf{P}$ is computable.

(3) Problem 3 for $\mathbf{P}$ is computable.

(4) The monadic theory of $\langle$ Nat, $<, \mathbf{P}\rangle$ is decidable.

(5) For every $M L O$ formula $\psi(X, Y, P)$ either there is a recursive $C$-operator $F$ such that $N a t \models \forall X \psi(X, F(X), \mathbf{P})$ or there is a recursive $S C$-operator $G$ such that $N a t \models$ $\forall Y \neg \psi(G(Y), Y, \mathbf{P})$. Moreover, it is decidable which of these cases holds and the (description of the) corresponding operator is computable from $\psi$.

The more difficult part of this theorem is the implication $(4) \Rightarrow(5)$.

The trivial examples of predicates with decidable monadic theory are ultimately periodic predicates. Recall that a predicate $\mathbf{P}$ is ultimately periodic if there are $0<p, d \in N a t$ such that $(n \in \mathbf{P} \leftrightarrow n+p \in \mathbf{P})$ for all $n>d$. Ultimately periodic predicates are $M L O-$ definable. Hence, for these predicates computability of Problems 1-5 can be derived from Theorem 1.1,

We prove that the Büchi-Landweber theorem can be extended only to ultimately periodic parameters.

Theorem 1.3. Let $\mathbf{P}$ be a subset of Nat. The following conditions are equivalent and imply computability of Problem 4: 
(1) $\mathbf{P}$ is ultimately periodic.

(2) For every $M L O$ formula $\psi(X, Y, P)$ either there is a finite state $C$-operator $F$ such that $N a t \models \forall X \psi(X, F(X, \mathbf{P}), \mathbf{P})$ or there is a finite state $S C$-operator $G$ such that $N a t \models \forall Y \neg \psi(G(Y, \mathbf{P}), Y, \mathbf{P})$.

In Problems 1-5 we restrict the computational complexity of the C-operators (implementations) which meet specifications. Another approach is to restrict their descriptive complexity. The finite state operators are $M L O$-definable in $\omega=\langle N a t,<\rangle$. An operator $F$ is defined by a formula $\psi(X, Y, P)$ in an expansion $M=\langle N a t,<, \mathbf{P}\rangle$ of $\omega$, if for all $\omega$-strings $\mathbf{X}$ and $\mathbf{Y}$ :

$$
\mathbf{Y}=F(\mathbf{X}) \text { iff } \omega \models \psi(\mathbf{X}, \mathbf{Y}, \mathbf{P})
$$

An operator $F$ is $M L O$-definable in $M=\langle N a t,<, \mathbf{P}\rangle$, if it is defined by an $M L O$ formula in $M$

Our main theorem which is stated in the next section implies

Theorem 1.4. For every $M L O$ formula $\psi(X, Y, P)$ and every expansion $M=\langle N a t,<, \mathbf{P}\rangle$ of $\omega$ either there is an $M L O$-definable (in $M) C$-operator $F$ such that $M \models \forall X \psi(X, F(X), P)$ or there is an $M L O$-definable (in $M$ ) SC-operator $G$ such that $M \models \forall Y \neg \psi(G(Y), Y, P)$. Moreover, formulas which define these operators are computable from $\psi$

The paper is organized as follows. In the next section games and their connections to the Church synthesis problem are discussed, the Büchi and Landweber theorem is rephrased in the game theoretical language, and our main definability result - Theorem 2.3 - which implies Theorem 1.4 is stated.

In Section 3, Theorem 1.2 is derived as a consequence of Theorem 2.3. In Section 4, standard definitions and facts about automata and logic are recalled. In Section 5 , finite state synthesis problems with parameters are considered and Theorem 1.3 is proved.

In Section 6. parity games on graphs and their connection to the synthesis problems are discussed and definability results needed in the proof of Theorem 2.3 are proved. The proof of Theorem 2.3 is given in Section 7.

Finally, in Section 8, some open problems are stated and further results are discussed.

\section{Game Version of the Church Problem and Main Definability Result}

Let $\mathbf{W}$ be a set of pairs of $\omega$ strings over $\{0,1\}$. A game $G(\mathbf{W})$ is defined as follows.

(1) The game is played by two players, called Player I (or Mr. X) and Player II (or Mr. $Y)$.

(2) A play of the game has $\omega$ rounds.

(3) At round $n$ : first, Player I chooses $x(n) \in\{0,1\}$; then, Player II chooses $y(n) \in\{0,1\}$.

(4) By the end of the play the $\omega$ strings $\mathbf{Y}=y(0) y(1) \ldots$ and $\mathbf{X}=x(0) x(1) \ldots$ over $\{0,1\}$ have been constructed.

Winning conditions: Player II wins the play if the pair of $\omega$-strings $\langle\mathbf{X}, \mathbf{Y}\rangle$ is in $\mathbf{W}$; otherwise, Player I wins the play.

What we want to know is: Does either one of the players have a winning strategy in $G(\mathbf{W})$. That is, can Player I choose his moves so that in whatever way Player II responds, we have $\langle\mathbf{X}, \mathbf{Y}\rangle \notin \mathbf{W}$ ? Or can Player II respond to Player I's moves in a way that ensures the opposite? 
Since at round $n$, Player I has access only to $\mathbf{Y} \cap[0, n)$ and Player II has access only to $\mathbf{X} \cap[0, n]$, a strategy of Player I (respectively, of Player II) is a strongly causal (respectively, causal) operator. So, a winning strategy for Player II is a causal operator $F:\{0,1\}^{\omega} \rightarrow\{0,1\}^{\omega}$ such that $\langle\mathbf{X}, F(\mathbf{X})\rangle$ is in $\mathbf{W}$ for every $\mathbf{X} \in\{0,1\}^{\omega}$, and a winning strategy for Player I is a strongly causal operator $G:\{0,1\}^{\omega} \rightarrow\{0,1\}^{\omega}$ such that $\langle G(\mathbf{Y}), \mathbf{Y}\rangle$ is not in $\mathbf{W}$ for every $\mathbf{Y} \in\{0,1\}^{\omega}$.

There is a natural topology on the set of $\omega$-strings (see e.g., PP04). According to this topology, subsets of $\omega$-strings can be classified as open, closed, Borel and so forth. The following theorem of Martin is fundamental.

Theorem 2.1 (Determinacy of Borel Games). For every Borel set $\mathbf{W}$, one of the players has a winning strategy in $G(\mathbf{W})$.

It was McNaughton (see [Mc66]) who first observed that the Church problem can be equivalently phrased in game-theoretic language. Algorithmic questions deal with finitely described objects. Hence, McNaughton considered games $G(\mathbf{W})$ only for definable sets $\mathbf{W}$.

Let $M=\langle$ Nat, $<, \mathbf{P}\rangle$ be a structure. A set $\mathbf{W} \subseteq\{0,1\}^{\omega} \times\{0,1\}^{\omega}$ is defined by an $M L O$ formula $\psi(X, Y, P)$ in $M$ if $\mathbf{W}=\{\langle\mathbf{X}, \mathbf{Y}\rangle: M \models \psi(\mathbf{X}, \mathbf{Y}, P)\}$. A set is $M L O$-definable iff it is defined by an $M L O$ formula. Similarly, an operator $F:\{0,1\}^{\omega} \rightarrow\{0,1\}^{\omega}$ is definable in $M$ if its graph $\{\langle\mathbf{X}, \mathbf{Y}\rangle: \mathbf{Y}=F(\mathbf{X})\}$ is definable; a strategy is definable iff the corresponding causal operator is definable.

Let $M=\left\langle N a t,<, \mathbf{P}_{1}, \ldots, \mathbf{P}_{n}\right\rangle$ be an expansion of $\omega=\langle N a t,<\rangle$ by unary predicates and let $\psi\left(X, Y, P_{1}, \ldots, P_{n}\right)$ be an $M L O$ formula. The McNaughton game $\mathcal{G}_{\psi}^{M}$ is the game $G(\mathbf{W})$, where $\mathbf{W}$ is the set definable by $\psi$ in $M$. Hence, the winning condition of $\mathcal{G}_{\psi}^{M}$ can be stated as

Winning conditions for $\mathcal{G}_{\psi}^{M}$ : Player II wins a play if $M \models \psi\left(\mathbf{X}, \mathbf{Y}, P_{1}, \ldots, P_{n}\right)$; otherwise, Player I wins the play.

This leads to

Game version of the Church problem: Let $M=\left\langle N a t,<, \mathbf{P}_{1}, \ldots, \mathbf{P}_{n}\right\rangle$ be an expansion of $\omega=\langle N a t,<\rangle$ by unary predicates. Given a formula $\psi\left(X, Y, P_{1}, \ldots, P_{n}\right)$ decide whether Player II has a winning strategy in $\mathcal{G}_{\psi}^{M}$.

Theorem 1.1 states the computability of the Church problem in the structure $\omega=$ $\langle N a t,<\rangle$ (no additional unary predicates). Even more importantly, Büchi and Landweber show that in the case of $\omega$ we can restrict ourselves to definable strategies, i.e., to causal (or strongly causal) operators computable by finite state automata or equivalently $M L O$-definable in $\omega$. The Büchi-Landweber theorem can be stated in the game theoretical language as follows:

Theorem 2.1 (Büchi-Landweber, 1969). Let $\psi(X, Y)$ be an MLO formula., Then:

Determinacy: One of the players has a winning strategy in the game $\mathcal{G}_{\psi}^{\omega}$.

Decidability: It is decidable which of the players has a winning strategy.

Definable strategy: The player who has a winning strategy also has an $M L O$-definable winning strategy.

Synthesis algorithm: We can compute a formula $\varphi(X, Y)$ that defines $($ in $(\omega,<))$ a winning strategy for the winning player in $\mathcal{G}_{\psi}^{\omega}$.

The determinacy part of the Büchi-Landweber theorem follows from the determinacy of Borel games. More generally, it is well known that for each $M L O$ formula $\psi\left(X, Y, Z_{1}, \ldots, Z_{n}\right)$ 
and $\mathbf{P}_{1}, \ldots, \mathbf{P}_{n} \subseteq$ Nat the set $\mathbf{W}=\left\{\langle\mathbf{X}, \mathbf{Y}\rangle:\langle N a t,<\rangle \models \psi\left(\mathbf{X}, \mathbf{Y}, \mathbf{P}_{1}, \ldots, \mathbf{P}_{n}\right)\right\}$ is a Borel set (it is even inside the boolean closure of the second level of the Borel hierarchy, see e.g., [PP04]). Hence, by the determinacy of Borel games, we obtain determinacy of the McNaughton games. In other words, we have the following corollary:

Corollary 2.2 (Determinacy). Let $M=\left\langle N a t,<, \mathbf{P}_{1}, \ldots, \mathbf{P}_{n}\right\rangle$ be an expansion of $\omega$ by unary predicates. Then, for every $M L O$ formula $\psi\left(X, Y, P_{1}, \ldots, P_{n}\right)$

(1) One of the players has a winning strategy in $\mathcal{G}_{\psi}^{M}$.

(2) Equivalently, either there is a C-operator $F$ such that $M \models \forall X \psi(X, F(X))$, or there is a $S C$-operator $G$ such that $M \models \forall Y \neg \psi(G(Y), Y)$.

In order to simplify notations, from now on, we will state our results only for the expansions of $\omega$ by one unary predicate. The generalization to the expansions by any number of unary predicates is straightforward.

Now, we are ready to state our main result which generalizes the definability and synthesis parts of the Büchi-Landweber theorem in a uniform way to the expansions of $\langle$ Nat,$<\rangle$ by unary predicates.

Theorem 2.3 (Main). There is an algorithm that given a formula $\varphi(X, Y, P)$ constructs a sentence $\operatorname{WIN}_{\varphi}^{I I}(P)$ and formulas $\operatorname{St}_{\varphi}^{I}(X, Y, P), S_{\varphi}^{I I}(X, Y, P)$ such that for every structure $M=\langle$ Nat,$<, \mathbf{P}\rangle$ Player II wins the games $\mathcal{G}_{\varphi}^{M}$ iff $M \models W I N_{\varphi}^{I I}$. Moreover, if Player II wins $\mathcal{G}_{\varphi}^{M}$, then $S_{\varphi}^{I I}(X, Y, P)$ defines his winning strategy; otherwise, St $t_{\varphi}^{I}$ defines a winning strategy of Player I.

Theorem 1.4 is reformulated in the game language as follows.

Theorem 2.4 (Game version of Theorem 1.4). For every $M L O$ formula $\psi(X, Y, P)$ and every expansion $M=\langle N a t,<, \mathbf{P}\rangle$ of $\omega$ :

Determinacy: One of the players has a winning strategy in the game $\mathcal{G}_{\psi}^{M}$.

Definable strategy: The player who has a winning strategy also has an MLO-definable (in $M$ ) winning strategy.

Synthesis algorithm: We can compute a formula that defines (in $M$ ) a winning strategy for the winning player in $\mathcal{G}_{\psi}^{M}$.

The determinacy part of Theorem 2.4 follows from Corollary 2.2, Its definability and synthesis parts are immediate consequences of Theorem 2.3. In the next section, Theorem 1.2 is derived as another consequence of Theorem 2.3 and of Corollary 2.2 .

The proof of Theorem 2.3 will be given in Sect. 7. Section 4 provides an additional background on logic and automata, and Section 6 prepares definability results needed in the proof of Theorem 2.3 .

\section{Proof of Theorem 1.2}

In this section we prove Theorem 1.2. Its more difficult part is the implication $(4) \Rightarrow(5)$; the proof of this implication is easily obtained from Theorem 2.3 . The proof of the other equivalences of Theorem 1.2 uses only determinacy and some simple facts.

We start with the following simple Lemma:

Lemma 3.1. Assume that the monadic theory of $M=\left\langle N a t,<, \mathbf{P}_{1}, \ldots, \mathbf{P}_{n}\right\rangle$ is decidable

(1) Every set $\mathbf{Q} \subseteq$ Nat definable in $M$ is recursive. 
(2) Every $C$-operator $F$ definable in $M$ is recursive.

(3) There is an algorithm that computes a program for $\mathbf{Q}$ (respectively, for $F$ ) from a formula which defines $\mathbf{Q}$ (respectively, $F$ ).

Proof. (1) Assume that $\mathbf{Q}$ is defined in $M$ by a formula $\psi(X)$. Each $j \in N a t$ is defined by an $M L O$ formula $\varphi_{j}(t)$. Hence, one can check whether $j \in \mathbf{Q}$, by testing whether the sentence $\exists X \exists t \varphi_{j}(t) \wedge \psi(X) \wedge t \in X$ holds in $M$.

(2) Assume that a $C$-operator $F$ is defined in $M$ by a formula $\psi(X, Y)$. We have to show that there is an algorithm which computes the $n$-th letter of $F(\mathbf{X})$ from the first $n$ letters of $\mathbf{X}$. Let $a=a_{0} \ldots a_{n} \in\{0,1\}^{*}$. Define $\zeta_{i}^{a}(t, X)$ as $\varphi_{i}(t) \wedge t \in X$ if $a_{i}$ is 1 , and as $\varphi_{i}(t) \wedge t \notin X$ otherwise, where $\varphi_{i}(t)$ is a formula which defines a number $i$. Note that $\left.M \models \exists t_{0} \exists t_{1} \ldots \exists t_{n} \bigwedge_{i=0}^{n} \zeta_{i}^{a}\left(t_{i}, \mathbf{X}\right)\right)$ iff the first $n+1$ letters of $\mathbf{X}$ are $a_{0} \ldots a_{n}$.

Now, if $a_{0} \ldots a_{n} \in\{0,1\}^{*}$ are the first $n+1$ letters of $\mathbf{X}$, then the $n+1$-th letter of $F(\mathbf{X})$ is 1 iff

$$
\exists X \exists Y \exists t_{0} \exists t_{1} \ldots \exists t_{n}\left(\psi(X, Y) \wedge \varphi_{n}\left(t_{n}\right) \wedge t_{n} \in Y \wedge \bigwedge_{i=0}^{n} \zeta_{i}^{a}\left(t_{i}, X\right)\right)
$$

holds in $M$.

(3) follows from the proofs of (1) and (2).

Lemma 3.2 (Implication $(4) \Rightarrow(5)$ of Theorem 1.2). Assume that the monadic theory of $M=\langle$ Nat, $<, \mathbf{P}\rangle$ is decidable. Then for every $M L O$ formula $\varphi(X, Y, P)$ either there is a recursive $C$-operator $F$ such that $N a t \models \forall X \varphi(X, F(X), \mathbf{P})$ or there is a recursive $S C$ operator $G$ such that $N a t \models \forall Y \neg \varphi(G(Y), Y, \mathbf{P})$. Moreover, it is decidable which of these cases holds and the (description of the) corresponding operator is computable from $\varphi$.

Proof. For a formula $\varphi$, construct $\operatorname{WIN}_{\varphi}^{I I}(P)$ and formulas $S t_{\varphi}^{I}(X, Y, P), S t_{\varphi}^{I I}(X, Y, P)$, as in Theorem 2.3.

By the assumption that the monadic theory of $M$ is decidable, we can check whether $W_{\varphi} N_{\varphi}^{I I}(P)$ holds in $M$.

If $\operatorname{WIN}_{\varphi}^{I I}(P)$ holds in $M$ then, by Theorem 2.3, $S t_{\varphi}^{I I}(X, Y, P)$ defines a winning strategy $F$ for Player II. Hence, $F$ is C-operator and $N a t \models \forall X \varphi(X, F(X), \mathbf{P})$. By Lemma 3.1, $F$ is recursive.

If $W I N_{\varphi}^{I I}(P)$ does not hold in $M$, then by Theorem 2.3, $S t_{\varphi}^{I}(X, Y, P)$ defines a winning strategy $G$ for Player I. Hence, $G$ is SC-operator and $N a t \models \forall Y \neg \varphi(G(Y), Y, \mathbf{P})$. Moreover, by Lemma 3.1, $G$ is recursive.

Lemma 3.3. If one of the Problems 1-5 is computable for $\mathbf{P}$, then the monadic theory of $\langle$ Nat,$<, \mathbf{P}\rangle$ is decidable.

Proof. Let $\beta(P)$ be a sentence in $M L O$ and let $\psi_{\beta}(X, Y, P)$ be defined as

$$
(\beta \rightarrow(Y=\{0\})) \wedge(\neg \beta \rightarrow(X=\emptyset)) .
$$

Observe that $N a t \models \beta(\mathbf{P})$ iff there is a C-operator $F$ such that:

$$
N a t \models \forall X \psi_{\beta}(X, F(X, \mathbf{P}), \mathbf{P}) \text { iff } N a t \models \forall X \psi_{\beta}(X, H(X, \mathbf{P}), \mathbf{P})
$$

where $H$ is a constant C-operator defined as $H=\lambda\langle X, P\rangle .10^{\omega}$.

Hence, if one of the Problems 1-5 is computable for $\mathbf{P}$, then we can decide whether Nat $\models \beta(\mathbf{P})$. 
The proof of Lemma 3.3 also implies that if the following Problem $1^{\prime}$ is decidable for $\mathbf{P}$, then the monadic theory of $\langle N a t,<, \mathbf{P}\rangle$ is decidable.

Decision Problem $1^{\prime}$ for $\mathbf{P} \subseteq N a t$
Input: an $M L O$ formulas $\psi(X, Y, P)$.
Question: Check whether there is a C-operator $Y=F(X, P)$ such that
$N a t \models \forall X \psi(X, F(X, \mathbf{P}), \mathbf{P})$.

Problem $1^{\prime}$ is actually Problem 1 without construction part.

Finally, we have

Lemma 3.4. The implications (5) $\Rightarrow(1),(5) \Rightarrow(2)$ and (5) $\Rightarrow(3)$ hold.

Proof. Let $\psi(X, Y, P)$ be a formula. By (5) either there is a recursive C-operator $F$ such that $N a t \models \forall X \psi(X, F(X), \mathbf{P})$ or there is a recursive SC-operator $G$ such that $N a t \models$ $\forall Y \neg \psi(G(Y), Y, \mathbf{P})$. Moreover, it is decidable which of these cases holds and the corresponding operator is computable from $\psi$.

In the first case, the answer to Problems 1-3 is positive and $F$ is a corresponding operator.

In the second case, the answer to Problems 1-3 is negative.

Indeed, for the sake of contradiction, assume that there is a C-operator (even nonrecursive) $F$ such that $N a t \models \forall X \psi(X, F(X, \mathbf{P}), \mathbf{P})$. Observe that $F$ is a C-operator and $G$ is a SC-operator. Hence, $H=\lambda X . G(F(X, \mathbf{P}))$ is a SC-operator. Every SC-operator has a fixed point. Let $\mathbf{X}_{0}$ be a fixed point of $H$ and let $\mathbf{Y}_{0}=F\left(\mathbf{X}_{0}, \mathbf{P}\right)$. Then we have: $\mathbf{X}_{0}=G\left(\mathbf{Y}_{0}\right)$. Therefore, we obtain

$$
\begin{array}{rll}
N a t \models \psi\left(\mathbf{X}_{0}, \mathbf{Y}_{0}, \mathbf{P}\right) & \text { because } & N a t \models \forall X \psi(X, F(X, \mathbf{P}), \mathbf{P}), \quad \text { and } \\
N a t \models \neg \psi\left(\mathbf{X}_{0}, \mathbf{Y}_{0}, \mathbf{P}\right) & \text { because } & N a t \models \forall Y \neg \psi(G(Y), Y, \mathbf{P}) .
\end{array}
$$

Contradiction.

\section{Background on Logic And Automata}

4.1. Notations and Terminology. We use $k, l, m, n, i$ for natural numbers; $N a t$ for the set of natural numbers and capital bold letters $\mathbf{P}, \mathbf{S}, \mathbf{R}$ for subsets of Nat. We identify subsets of a set $A$ and the corresponding unary (monadic) predicates on $A$.

The set of all (respectively, non-empty) finite strings over an alphabet $\Sigma$ is denoted by $\Sigma^{*}$ (respectively, by $\Sigma^{+}$). The set of $\omega$-strings over $\Sigma$ is denoted by $\Sigma^{\omega}$.

Let $a_{0} \ldots a_{k} \ldots$ and $b_{0} \ldots b_{k} \ldots$ be $\omega$-strings. We say that these $\omega$-strings coincide on an interval $[i, j]$ if $a_{k}=b_{k}$ for $i \leq k \leq j$. A function $F$ from $\Sigma_{1}^{\omega}$ to $\Sigma_{2}^{\omega}$ will be called an operator of type $\Sigma_{1} \rightarrow \Sigma_{2}$. An operator $F$ is called causal (respectively, strongly causal) operator, if $F(X)$ and $F(Y)$ coincide on an interval $[0, t]$, whenever $X$ and $Y$ coincide on $[0, t]$ (respectively, on $[0, t)$ ). We will refer to causal (respectively, strongly causal) operators as C-operators (respectively, SC-operators).

Let $\Sigma_{1}$ and $\Sigma_{2}$ be finite alphabet and let $F: \Sigma_{1}^{\omega} \rightarrow \Sigma_{2}^{\omega}$ be a C-operator. Note that there is a unique function $h_{F}: \Sigma_{1}^{*} \rightarrow \Sigma_{2}$ such that $F\left(a_{1} \ldots a_{n}\right)=b_{n}$ if for some (equivalently for all) $\omega$-string $y: b_{n}$ is the $n$-th letter of $F\left(a_{1} \ldots a_{n} y\right) . F$ is said to be recursive if $h_{F}$ is recursive.

Every SC-operator $F$ of type $\Sigma \rightarrow \Sigma$ has a unique fixed point, i.e., there is a unique $X \in \Sigma^{\omega}$ such that $X=F(X)$. 
Let $G: \Sigma^{\omega} \rightarrow \Delta^{\omega}$ be an operator. In the case $\Sigma$ is the Cartesian product $\Sigma_{1} \times \Sigma_{2}$ we will identify $G$ with the corresponding operator $F: \Sigma_{1}^{\omega} \times \Sigma_{2}^{\omega} \rightarrow \Delta^{\omega}$. An operator $F: \Sigma_{1}^{\omega} \times \Sigma_{2}^{\omega} \rightarrow \Delta^{\omega}$ is said to be $\mathrm{SC}$-operator (C-operator) if $G$ is $\mathrm{SC}$-operator (respectively, C-operator).

There exists a one-one correspondence between the set of all $\omega$-strings over the alphabet $\{0,1\}^{n}$ and the set of all $n$-tuples $\left\langle\mathbf{P}_{1}, \ldots, \mathbf{P}_{n}\right\rangle$ of unary predicates over the set of natural numbers. With an $n$-tuple $\left\langle\mathbf{P}_{1}, \ldots, \mathbf{P}_{n}\right\rangle$ of unary predicates over $N a t$, we associate the $\omega$-string $a_{0} a_{1} \ldots a_{k} \ldots$ over alphabet $\{0,1\}^{n}$ defined by $a_{k}={ }_{\text {def }}\left\langle b_{1}^{k}, \ldots b_{n}^{k}\right\rangle$ where $b_{i}^{k}$ is 1 if $\mathbf{P}_{i}(k)$ holds and $b_{i}^{k}$ is 0 otherwise. Let $Q=\left\{q_{1}, \ldots, q_{m}\right\}$ be a finite set of state. There is a natural one-one correspondence between the subsets of $Q \times N a t$ and the set of $m$-tuples of unary predicates over $N a t$ : with $U \subseteq Q \times N a t$ we associate the $m$-tuple $\left\langle\mathbf{P}_{1}, \ldots, \mathbf{P}_{m}\right\rangle$ defined as $i \in \mathbf{P}_{j}$ iff $U\left(q_{j}, i\right)$ (for $i \in N a t$ and $j \leq m$ ).

Similarly, there is a one-one correspondence between the set of all strings of length $m$ over the alphabet $\{0,1\}^{n}$ and the set of all $n$-tuples $\left\langle\mathbf{P}_{1}, \ldots, \mathbf{P}_{n}\right\rangle$ of unary predicates over the set $\{0, \ldots, m-1\}$.

A linearly ordered set will be called a chain. A chain with $n$ monadic predicates over its domain will be called an $n$-labelled chain; whenever $n$ is clear from the context, $n$-labelled chains will be called labelled chains.

We will sometimes identify an $n$-labelled chain $M=\left\langle N a t,<, \mathbf{P}_{1}, \ldots, \mathbf{P}_{n}\right\rangle$ with the $\omega$ string over the alphabet $\{0,1\}^{n}$ which corresponds to the $n$-tuple $\left\langle\mathbf{P}_{1}, \ldots, \mathbf{P}_{n}\right\rangle$; this $\omega$-string will be called the characteristic $\omega$-string (or $\omega$-word) of $M$. Similarly, we will identify finite $n$-labelled chains with corresponding strings over $\{0,1\}^{n}$.

4.2. Monadic Second-Order Logic and Monadic Logic of Order. Let $\sigma$ be a relational signature. Atomic formulas of the monadic second-order logic over $\sigma$ are $R\left(t_{1}, \ldots, t_{n}\right)$, $t_{1}=t_{2}$, and $t_{1} \in X$ where $t_{1}, \ldots, t_{n}$ are individual variables, $R \in \sigma$ is an n-are relational symbol, and $X$ is a set variable. Formulas are obtained from atomic formulas by conjunction, negation, and quantification $\exists t$ and $\exists X$ for $t$ an individual and $X$ a set variable. The satisfaction relation $M, \tau_{1}, \ldots \tau_{k} ; \mathbf{S}_{1}, \ldots, \mathbf{S}_{m} \models \varphi\left(t_{1}, \ldots, t_{k} ; X_{1}, \ldots, X_{m}\right)$ is defined as usual with the understanding that set variables range over subsets of $M$.

We use standard abbreviations, e.g., we write $X \subseteq X^{\prime}$ for $\forall t . X(t) \rightarrow X^{\prime}(t)$; we write $X=X^{\prime}$ for $\forall t . X(t) \leftrightarrow X^{\prime}(t)$; symbols " $\exists \leq 1$ " and " $\exists$ !" stands for "there is at most one" and "there is a unique".

If a signature $\sigma$ contains one binary predicate $<$ which is interpreted as a linear order, and all other predicates are unary, the monadic second-order logic for this signature is called Monadic Logic of Order $(M L O)$. The formulas of $M L O$ are interpreted over labelled chains.

The monadic theory of a labelled chain $M$ is the set of all $M L O$ sentences which hold in $M$.

We will deal with the expansions of $\omega$ by monadic predicates, i.e., with the structures of the form $M=\left\langle N a t,<, \mathbf{P}_{1}, \ldots, \mathbf{P}_{n}\right\rangle$. We say that a chain $M=\left\langle N a t,<, \mathbf{P}_{1}, \ldots, \mathbf{P}_{n}\right\rangle$ is recursive if all $\mathbf{P}_{i}$ are recursive subsets of $N a t$.

An $\omega$-language $L$ is said to be defined by an $M L O$ formula $\psi\left(X_{1}, \ldots, X_{n}\right)$ if the following condition holds: an $\omega$ string is in $L$ iff the corresponding $n$-tuple of unary predicates satisfies $\psi$. 
4.3. The First-Order Version of the Monadic Second-Order Logic. Sometimes it will be convenient for us to consider the first-order version of the monadic second order logic.

Let $\sigma$ be a relational signature and $M$ be a structure for $\sigma$.

Let $\bar{\sigma}=\sigma \cup\{$ Sing,$\subseteq\}$ where Sing is a new unary relational symbol and $\subseteq$ a new binary relational symbol. Let $\bar{M}$ be the structure for $\bar{\sigma}$ defined as follows: The domain of $\bar{M}$ is the set of all subsets of the domain of $M$. $\operatorname{Sing}(A)$ holds in $\bar{M}$ if $A$ is one element subset; $A \subseteq B$ holds if $A$ is a subset of $B$; for $k$-ary relational symbol $R \in \sigma$ :

$$
\begin{gathered}
R\left(A_{1}, \ldots A_{k}\right) \text { holds in } \bar{M} \text { iff } \\
A_{1}=\left\{a_{1}\right\}, \ldots, A_{k}=\left\{a_{k}\right\} \text { and } R\left(a_{1}, \ldots a_{k}\right) \text { holds in } M
\end{gathered}
$$

The following lemma is well-known and is easily proved by the structural induction.

Lemma 4.1 (Equivalence of two Versions of Monadic Logic). The two versions of Monadic logic are expressive equivalent, that is

(1) Let $\psi\left(X_{1}, \ldots, X_{k}\right)$ be a formula of the monadic second-order logic for a signature $\sigma$. There is a first-order formula $\varphi\left(X_{1}, \ldots, X_{k}\right)$ in the signature $\bar{\sigma}$ such that for every structure $M$ for the signature $\sigma$ and for subsets $A_{1}, \ldots, A_{k}$ of the domain of $M$

$$
M, A_{1}, \ldots, A_{k} \models \psi\left(X_{1}, \ldots, X_{k}\right) \text { iff } \bar{M}, A_{1}, \ldots, A_{k} \models \varphi\left(X_{1}, \ldots, X_{k}\right) .
$$

Moreover there is an algorithm that computes $\varphi$ from $\psi$.

(2) Let $\varphi\left(X_{1}, \ldots, X_{k}\right)$ be a first-order formula in the signature $\bar{\sigma}$. There is a formula in the monadic second-order logic for the signature $\sigma$ such that for every structure $M$ for the signature $\sigma$ and for subsets $A_{1}, \ldots, A_{k}$ of the domain of $M$

$$
M, A_{1}, \ldots, A_{k} \models \psi\left(X_{1}, \ldots, X_{k}\right) \text { iff } \bar{M}, A_{1}, \ldots, A_{k} \models \varphi\left(X_{1}, \ldots, X_{k}\right) .
$$

Moreover there is an algorithm that computes $\psi$ from $\varphi$.

4.4. Automata. A deterministic transition system $D$ is a tuple $\left\langle\mathcal{Q}, \Sigma, \delta, q_{\text {init }}\right\rangle$, consisting of a set $\mathcal{Q}$ of states, an alphabet $\Sigma$, a transition function $\delta: \mathcal{Q} \times \Sigma \rightarrow \mathcal{Q}$ and initial state $q_{\text {init }} \in \mathcal{Q}$. The transition function is extended as usual to a function from $\mathcal{Q} \times \Sigma^{*}$ to $\mathcal{Q}$ which will be also denoted by $\delta$. The function $\delta_{\text {init }}: \Sigma^{*} \rightarrow \mathcal{Q}$ is defined as $\delta_{\text {init }}(\pi)=\delta\left(q_{\text {init }}, \pi\right)$. A transition systems is finite if $\mathcal{Q}$ and $\Sigma$ are finite.

A finite deterministic automaton $\mathcal{A}$ is a tuple $\left\langle\mathcal{Q}, \Sigma, \delta, q_{\text {init }}, F\right\rangle$, where $\left\langle\mathcal{Q}, \Sigma, \delta, q_{\text {init }}\right\rangle$ is a finite deterministic transition system and $F$ is a subset of $\mathcal{Q}$. A string $\pi \in \Sigma^{*}$ is accepted by $\mathcal{A}$ if $\delta_{\text {init }}(\pi) \in F$. The language accepted (or defined) by $\mathcal{A}$ is the set of string accepted by $\mathcal{A}$.

A Mealey automaton is a tuple $\left\langle\mathcal{Q}, \Sigma, \delta, q_{\text {init }}, \Delta\right.$, out $\rangle$, where $\left\langle\mathcal{Q}, \Sigma, \delta, q_{\text {init }}\right\rangle$ is a deterministic transition system, $\Delta$ is an alphabet and out $: \mathcal{Q} \rightarrow \Delta$ is an output function. With a Mealey automaton $\mathcal{A}=\left\langle\mathcal{Q}, \Sigma, \delta, q_{\text {init }}, \Delta\right.$, out $\rangle$ we associate a function $h_{\mathcal{A}}: \Sigma^{*} \rightarrow \Delta$ and an operator $F_{\mathcal{A}}: \Sigma^{\omega} \rightarrow \Delta^{\omega}$ defined as follows:

$$
\begin{gathered}
h_{\mathcal{A}}\left(a_{0} \ldots a_{i-1}\right)=\operatorname{out}\left(\delta_{\text {init }}\left(a_{0} \ldots a_{i-1}\right)\right) \\
F_{\mathcal{A}}\left(a_{0} \ldots a_{i} \ldots\right)=b_{0} \ldots b_{i} \ldots \text { iff } b_{i}=h_{\mathcal{A}}\left(a_{0} \ldots a_{i-1}\right)
\end{gathered}
$$

It is easy to see that an operator is strongly causal (SC-operator) iff it is definable by a Mealey automaton. We say that a SC-operator $F: \Sigma^{\omega} \rightarrow \Delta^{\omega}$ is finite state iff it is definable by a finite state Mealey automaton. 
A finite Mealey automaton $\mathcal{A}=\left\langle\mathcal{Q}, \Sigma, \rightarrow, \delta, q_{\text {init }}, \Delta, \operatorname{col}\right\rangle$, where the output alphabet $\Delta$ is a (finite) subset of $N a t$, is called a (deterministic) parity automaton; the output function col is usually refered to as coloring function.

With every $\omega$-string $a_{0} a_{1} \cdots a_{i} \cdots \in \Sigma^{\omega}$ we associate the $\omega$-sequence of successive states $\delta_{\text {init }}\left(a_{0}\right) \delta_{\text {init }}\left(a_{0} a_{1}\right) \cdots \delta_{\text {init }}\left(a_{0} \cdots a_{i}\right) \cdots$ and the set Inf of all $q \in \mathcal{Q}$ that appear infinitely many times in this sequence. An $\omega$-string is accepted by $\mathcal{A}$ if the minimal element of the set $\{\operatorname{col}(q): q \in \operatorname{Inf}\}$ is even. The $\omega$-language accepted (or defined) by $\mathcal{A}$ is the set of all $\omega$-strings accepted by $\mathcal{A}$.

Sometimes the alphabet $\Sigma$ of $\mathcal{A}$ will be the Cartesian product $\Sigma_{1} \times \Sigma_{2} \times \Sigma_{3}$ of other alphabets. In this case we say that $\mathcal{A}$ defines a relation $R_{\mathcal{A}} \subseteq \Sigma_{1}^{\omega} \times \Sigma_{2}^{\omega} \times \Sigma_{3}^{\omega}$; a triplet $\langle a, b, c\rangle$ of $\omega$-strings is in $R_{\mathcal{A}}$ iff the $\omega$ string $\left(a_{0}, b_{0}, c_{0}\right)\left(a_{1}, b_{1}, c_{1}\right) \ldots\left(a_{i}, b_{i}, c_{i}\right) \ldots$ is accepted by $\mathcal{A}$.

Here is the classical theorem due to Büchi, Elgot and Trakhtenbrot.

Theorem 4.1. (1) A language is accepted by a finite deterministic automaton iff it is definable by an $M L O$ formula.

(2) An w-language is accepted by a deterministic parity automaton iff it is definable by an $M L O$ formula.

(3) Moreover, there is an algorithm which for every formula $\varphi\left(X_{1}, \ldots, X_{m}\right)$ computes an equivalent deterministic automaton $\mathcal{A}$ i.e., the language definable by $\varphi$ is accepted by $\mathcal{A}$. There is an algorithm which for every deterministic automaton $\mathcal{A}$ computes an equivalent $M L O$ formula. Similarly, there are translation algorithms between formulas and deterministic parity automata.

A Moore automaton is a tuple $\left\langle\mathcal{Q}, \Sigma, \delta, q_{\text {init }}, \Delta\right.$, out $\rangle$, where $\left\langle\mathcal{Q}, \Sigma, \delta, q_{\text {init }}\right\rangle$ is a deterministic transition system, $\Delta$ is an alphabet and out : $\mathcal{Q} \times \Sigma \rightarrow \Delta$ is an output function.

With a Moore automaton $\mathcal{A}=\left\langle\mathcal{Q}, \Sigma, \delta, q_{\text {init }}, \Delta\right.$, out $\rangle$ we associate a function $h_{\mathcal{A}}: \Sigma^{+} \rightarrow \Delta$ and an operator $F_{\mathcal{A}}: \Sigma^{\omega} \rightarrow \Delta^{\omega}$ defined as follows:

$$
\begin{gathered}
h_{\mathcal{A}}\left(a_{0} \ldots, a_{i}\right)=\operatorname{out}\left(\delta_{\text {init }}\left(a_{0} \ldots a_{i-1}\right), a_{i}\right) \\
F_{\mathcal{A}}\left(a_{0} \ldots a_{i} \ldots\right)=b_{0} \ldots b_{i} \ldots \text { iff } b_{i}=h_{\mathcal{A}}\left(a_{0} \ldots, a_{i}\right)
\end{gathered}
$$

It is easy to see that an operator is causal (C-operator) iff it is definable by a Moore automaton.

We say that a C-operator $F: \Sigma^{\omega} \rightarrow \Delta^{\omega}$ is finite state iff it is definable by a finite state Moore automaton.

\section{Finite State Synthesis Problems with Parameters}

Recall that a predicate $\mathbf{P} \subseteq$ Nat is ultimately periodic if there is $p, d \in$ Nat such that $(n \in \mathbf{P} \leftrightarrow n+p \in \mathbf{P})$ for all $n>d$. Ultimately periodic predicates are $M L O$-definable. Therefore, for every ultimately periodic predicate $\mathbf{P}$ the monadic theory of $\left\langle N a t,{ }_{1} \mathbf{P}\right\rangle$ is decidable.

The next theorem implies Theorem 1.3 and shows that Theorem 1.1 can be extended only to ultimately periodic predicates.

Theorem 5.1. Let $\mathbf{P}$ be a subset of Nat. The following conditions are equivalent and imply computability of Problem 4:

(1) $\mathbf{P}$ is ultimately periodic. 
(2) For every $M L O$ formula $\psi(X, Y, P)$ either there is a finite state $C$-operator $F$ such that $N a t \models \forall X \psi(X, F(X, \mathbf{P}), \mathbf{P})$ or there is a finite state $C$-operator $G$ such that $N a t \models$ $\forall Y \neg \psi(G(Y, \mathbf{P}), Y, \mathbf{P})$.

(3) $\mathbf{P}$ satisfies the following selection condition:

For every formula $\alpha(X, P)$ such that $N a t \models \exists X \alpha(X, \mathbf{P})$ there is a finite state

$C$-operator $H:\{0,1\}^{\omega} \rightarrow\{0,1\}^{\omega}$ such that $N a t \models \alpha(H(\mathbf{P}), \mathbf{P})$.

Proof. The implication $(1) \Rightarrow(2)$ follows from Theorem 1.1 and the fact that every ultimately periodic predicate is definable by an $M L O$ formula. The implication $(2) \Rightarrow(3)$ is trivial.

The implication $(3) \Rightarrow(1)$ is derived as follows. Let $\alpha(X, P)$ be $\forall t(X(t) \leftrightarrow P(t+1))$. Note $N a t \models \exists X \alpha(X, \mathbf{P})$ for every $\mathbf{P} \subseteq N a t$. Therefore, if $\mathbf{P}$ satisfies selection condition, then there is C-operator $H:\{0,1\}^{\omega} \rightarrow\{0,1\}^{\omega}$ such that $N a t \models \alpha(H(\mathbf{P}), \mathbf{P})$.

Assume that a finite state Moore automaton $\mathcal{A}$ computes $H$ and has $n$ states. We are going to show that $\mathbf{P}$ is ultimately periodic with period at most $2 n+1$. For $i \in N a t$ let $a_{i}$ be one if $i \in \mathbf{P}$ and $a_{i}$ be zero otherwise. Let $q_{0} q_{1} \ldots q_{2 n+1} \ldots$ be the sequence states passed by $\mathcal{A}$ on the input $a_{0} a_{1} \ldots a_{2 n+1} \ldots$ There are $i<j<2 n$ such that $a_{i}=a_{j}$ and $q_{i}=q_{j}$. Observe that $q_{i+1}=\delta_{\mathcal{A}}\left(q_{i}, a_{i}\right)=\delta_{\mathcal{A}}\left(q_{j}, a_{j}\right)=q_{j+1}$ and $a_{i+1}=\operatorname{out}_{\mathcal{A}}\left(q_{i}, a_{i}\right)=$ $\operatorname{out}_{\mathcal{A}}\left(q_{j}, a_{j}\right)=a_{j+1}$. And by induction we get that $q_{i+m}=q_{j+m}$ and $a_{i+m}=a_{j+m}$ for all $m \in$ Nat. Therefore, $\mathbf{P}$ is an ultimately periodic with a period $j-i<2 n$.

Note that this theorem does not imply that Problem 4 is computable only for ultimately periodic predicates. The next theorem can be established by the same arguments.

Theorem 5.2. The following conditions are equivalent and imply computability of Problem 5:

(1) $\mathbf{P}$ is ultimately periodic.

(2) For every $M L O$ formula $\psi(X, Y, P)$ either there is a finite state $C$-operator $F$ such that $N a t \models \forall X \psi(X, F(X), \mathbf{P})$ or there is a finite state $S C$-operator $G$ such that $N a t \models$ $\forall Y \neg \psi(G(Y), Y, \mathbf{P})$. Moreover, it is decidable which of these cases holds and the corresponding operator is computable from $\psi$.

\section{Parity Games on Graphs and the Synthesis Problem}

In subsection 6.1, we provide standard definitions and facts about infinite two-player perfect information games on graphs. In [BL69, a reduction of the Church synthesis problem to infinite two-player games on finite graphs was provided. In subsection 6.2, we provide a reduction of the Church synthesis problem with parameters to infinite two-player games on infinite graphs; this reduction is "uniform" in the parameters. The main definability results needed for the proof of Theorem 2.3 are given in Sect. 6.3 and Sect. 6.4,

6.1. Parity Games on Graphs. We consider here two-player perfect information games, played on graphs, in which each player chooses, in turn, a vertex adjacent to a current vertex. The presentation is based on [PP04.

A (directed) bipartite graph $G=\left(V_{1}, V_{2}, E\right)$ is called a game arena if the outdegree of every vertex is at least one. If $G$ is an arena, a game on $G$ is defined by an initial node $v_{\text {init }} \in V_{1}$ and a set of winning $\omega$-paths $\mathcal{F}$ from this node. 
Player I plays on vertices in $V_{1}$ and Player II on vertices in $V_{2}$. A play from a node $v_{1}=v_{\text {init }}$ is an infinite path $v_{1} v_{2} \ldots v_{i} \ldots$ in $G$ formed by the two players starting from the initial position $v_{1}$. Whenever the current position $v_{i}$ belongs to $V_{1}$ (respectively $V_{2}$ ), then Player I (respectively, Player II) chooses a successor node $v_{i+1}$ such $E\left(v_{i}, v_{i+1}\right)$. Since the graph is bipartite, Player I plays at the odd positions $\left(v_{2 i+1} \in V_{i}\right)$ and Player II plays at the even positions $\left(v_{2 i} \in V_{2}\right)$. Player I wins if the play belongs to $\mathcal{F}$.

A strategy $f$ for Player I (Player II) is a function which assigns to every path of even (respectively, odd) length a node adjacent to the last node of the path. A play $v_{\text {init }} v_{2} v_{3} \ldots$ is played according to a strategy $f_{1}$ of Player I (strategy $f_{2}$ of Player II) if for every prefix $\pi=v_{\text {init }} v_{2} \ldots v_{n}$ of even (respectively, odd) length $v_{n+1}=f_{1}(\pi)$ (respectively, $v_{n+1}=f_{2}(\pi)$ ). A strategy is winning for Player I (respectively, for Player II) if all the plays played according to this strategy are in $\mathcal{F}$ (respectively, in the complement of $\mathcal{F}$ ). A strategy is memoryless if it depends only on the last nodes in the path.

Parity games are games on graphs in which the set of winning paths are defined by parity conditions. More precisely, let $G=\left(V_{1}, V_{2}, E\right)$ be a game arena and let $c: V_{1} \cup$ $V_{2} \rightarrow\{0,1, \ldots m\}$ be a coloring.

Let $\rho=v_{1} v_{2} \ldots$ be a play. With such a play $\rho$, we associate the set of colors $C_{\rho}$ that appear infinitely many times in the $\omega$-sequence $\operatorname{col}\left(v_{1}\right) \operatorname{col}\left(v_{2}\right) \ldots$; a play $\rho$ is winning for Player I if the minimal element of $C_{\rho}$ is odd. The following theorem due to Emerson/Julta and Mostowski (see, EJ91, GTW02, PP04]) is fundamental:

Theorem 6.1. In a parity game, one of the players has a memoryless winning strategy.

6.2. Games and the Church Synthesis Problem. Let $\mathcal{A}=\left\langle\mathcal{Q}, \Sigma, \delta_{\mathcal{A}}, q_{\text {init }}\right.$, col $\rangle$ be a deterministic parity automaton over the alphabet $\Sigma=\{0,1\} \times\{0,1\} \times\{0,1\}$, let $R_{\mathcal{A}} \subseteq$ $\{0,1\}^{\omega} \times\{0,1\}^{\omega} \times\{0,1\}^{\omega}$ be the relation defined by $\mathcal{A}$ and let $\mathbf{P}$ be a subset of $N a t$. We will define a parity game $G_{\mathcal{A}, \mathbf{P}}$ such that

(1) Player I has a winning strategy in $G_{\mathcal{A}, \mathbf{P}}$ iff there is a SC-operator $G:\{0,1\}^{\omega} \rightarrow\{0,1\}^{\omega}$ such that $\neg R_{\mathcal{A}}(G(Y), Y, \mathbf{P})$ holds for every $Y$.

(2) Player II has a winning strategy in $G_{\mathcal{A}, \mathbf{P}}$ iff there is a C-operator $F:\{0,1\}^{\omega} \rightarrow\{0,1\}^{\omega}$ such that $R_{\mathcal{A}}(X, F(X), \mathbf{P})$ holds for every $X$.

The arena $G\left(V_{1}, V_{2}, E\right)$ of $G_{\mathcal{A}, \mathbf{P}}$ is defined as follows:

Nodes: $V_{1}=\mathcal{Q} \times N a t$ and $V_{2}=\mathcal{Q} \times\{0,1\} \times N a t$.

Edges from $V_{1}$ : From $\langle q, n\rangle \in V_{1}$ two edges exit; one to $\langle q, 0, n\rangle \in V_{2}$, and the second to $\langle q, 1, n\rangle \in V_{2}$. We will assign labels to these edges. The first one will be labeled by 0 and the second one will be labeled by 1 . These edge labels play no role in the game on our graph; however, it will be convenient to refer to them later.

Edges from $V_{2}$ : From $\langle q, a, n\rangle \in V_{2}$ two edges exit defined as follows: let $c$ be 1 if $n \in \mathbf{P}$ and 0 if $n \notin \mathbf{P}$; and for $b \in\{0,1\}$ let $q_{b}$ be $\delta_{\mathcal{A}}(q,\langle a, b, c\rangle)$. One edge from $\langle q, a, n\rangle$ is connected to $\left\langle q_{0}, n+1\right\rangle$, and the second one to $\left\langle q_{1}, n+1\right\rangle$. We label the first edge by 0 , and the second one by 1 .

The color of a node of the arena is defined by the color of its automaton's component, i.e., $c(\langle q, n\rangle)=c(\langle q, a, n\rangle=\operatorname{col}(q)$.

The node $\left\langle q_{\text {init }}, 0\right\rangle$ is the initial node of the game.

Every node of the game graph for $G_{\mathcal{A}, \mathbf{P}}$ has two successors. The subsets of $V_{1}$ (respectively, of $V_{2}$ ) can be identified with the memoryless strategies of Player I (respectively, of 
Player II). For a subset $U_{1} \subseteq V_{1}$, the corresponding memoryless strategy $f_{U_{1}}$ is defined as

$$
f_{U_{1}}(\langle q, n\rangle)= \begin{cases}\langle q, 1, n\rangle & \text { if }\langle q, n\rangle \in U_{1} \\ \langle q, 0, n\rangle & \text { otherwise }\end{cases}
$$

In other words, for $v \in V_{1}$ the strategy $f_{U_{1}}$ chooses the nodes reachable from $v$ by the edge with the label $U_{1}(v)$.

Observation 6.1 (bijection between the memoryless strategies and the subset of nodes). The function that assigns to every subset $U$ of $V_{1}$ the strategy $f_{U}$ for Player I is a bijection between the set of memoryless strategies for Player I and the subset of $V_{1}$. Similarly, the function that assigns to every subset $U$ of $V_{2}$ the strategy $f_{U}$ is a bijection between the set of memoryless strategies for Player II and the subset of $V_{2}$.

A subset $U_{1} \subseteq V_{1}$, induces a function $h_{U_{1}}:\{0,1\}^{*} \rightarrow V_{2}$ and a SC-operator $F_{U_{1}}$ : $\{0,1\}^{\omega} \rightarrow\{0,1\}^{\omega}$. First, we provide the definition for $h_{U_{1}}$, and later for $F_{U_{1}}$.

Let $G_{U_{1}}$ be the subgraph of $G_{\mathcal{A}, \mathbf{P}}$, obtained by removing from every node $v \in V_{1}$ the edge labelled by $\neg U_{1}(v)$, and removing the label from the other edge exiting $v$. In this graph, every $V_{1}$ node has outdegree one, and every $V_{2}$ node has two exiting edges; one is labeled by 0 and the other is labeled by 1 . For every $\pi$ in $\{0,1\}^{*}$ there is a unique path from $\left\langle q_{\text {init }}, 0\right\rangle$ to a state $v_{2} \in V_{2}$ such that $\pi$ is the sequence of labels on the edges of this path; this node $v_{2}$ is $h_{U_{1}}$ image of $\pi$.

Now a SC-operator $F_{U_{1}}:\{0,1\}^{\omega} \rightarrow\{0,1\}^{\omega}$ induced by $U_{1}$ is defined as follows. Let $\pi=b_{0} b_{1} \ldots$ be an $\omega$-string. There is a unique $\omega$-path $\rho$ from $\left\langle q_{\text {init }}, 0\right\rangle$ in $G_{U_{1}}$ such that $\pi$ is the sequence labels on the edges of this path. Let $v_{1} v_{2} \ldots$ be the sequence of $V_{1}$ nodes on $\rho$ and let $a_{i}=1$ if $v_{i} \in U_{1}$ and 0 otherwise. The $\omega$ sequence $a_{0} a_{1} \ldots$ is defined as the $F_{U_{1}}$ image of $\pi$.

Similarly, $U_{2} \subseteq V_{2}$ induces a function $h_{U_{2}}:\{0,1\}^{+} \rightarrow V_{1}$ and C-operator $F_{U_{2}}$.

Below we often use "a function $F$ corresponds to a set $U$ " as synonym "a set $U$ induces a function $F^{\prime \prime}$.

The properties of the above constructions are summarized as follows:

Lemma 6.2. (1) Let $U_{1}$ be a subset of $V_{1}$. The memoryless strategy defined by $U_{1}$ is winning for Player I in $G_{\mathcal{A}, \mathbf{P}}$ iff $\neg R_{\mathcal{A}}\left(F_{U_{1}}(Y), Y, \mathbf{P}\right)$ holds for every $Y$.

(2) Let $U_{2}$ be a subset of $V_{2}$. The memoryless strategy defined by $U_{2}$ is winning for Player II in $G_{\mathcal{A}, \mathbf{P}}$ iff $R_{\mathcal{A}}\left(X, F_{U_{2}}(X), \mathbf{P}\right)$ holds for every $X$.

(3) Let $\varphi(X, Y, Z)$ be an $M L O$ formula equivalent to $\mathcal{A}$, let $\psi$ be $\varphi(X, Y, P)$ and let $M$ be $\langle$ Nat,$<, \mathbf{P}\rangle$. The memoryless strategy defined by $U$ is winning for Player I (respectively, Player II) in $G_{\mathcal{A}, \mathbf{P}}$ iff the operator induced by $U$ is a winning strategy for Player $I$ (respectively, for Player II) in $\mathcal{G}_{\psi}^{M}$.

Our next objective is to show that the set of memoryless winning strategies and the operator induced by a memoryless strategy are $M L O$-definable in $\langle N a t,<, \mathbf{P}\rangle$. For this purpose, we show in 6.3 that these are definable in the monadic-second order logic for the structure appropriate for the game graph $G_{\mathcal{A}, \mathbf{P}}$. Then, in Sect. 6.4, we translate these definitions to $M L O$ formulas over $\langle$ Nat, $<, \mathbf{P}\rangle$.

6.3. Definability in the game structure. The game arena $G_{\mathcal{A}, \mathbf{P}}$ can be considered as a logical structure $M=M_{\mathcal{A}, \mathbf{P}}$ for the signature $\tau_{\mathcal{A}}=\left\{R_{i}: i \in \mathcal{Q} \cup \mathcal{Q} \times\{0,1\}\right\} \cup\{$ Init $, P, \prec$ 
, $\left.E_{0}, E_{1}\right\}$, where $R_{i}$, Init and $P$ are unary predicates and $\prec,, E_{0}, E_{1}$ are binary predicates with the interpretation

$$
\begin{aligned}
& R_{i}^{M}= \begin{cases}\{\langle q, j\rangle: j \in N a t\} & \text { for } i=q \in \mathcal{Q} \\
\{\langle q, a, j\rangle: j \in N a t\} & \text { for } i=\langle q, a\rangle \in \mathcal{Q} \times\{0,1\}\end{cases} \\
& P^{M}=\{\langle q, m\rangle: m \in \mathbf{P}\} \cup\{\langle q, a, m\rangle: a \in\{0,1\} \text { and } m \in \mathbf{P}\} \\
& \text { Init }^{M}=\left\{\left\langle q_{\text {init }}, 0\right\rangle\right\} \\
& \left.\mathbf{E}_{0}^{M}\left(v_{1}, v_{2}\right) \text { (respectively, } \mathbf{E}_{1}^{M}\left(v_{1}, v_{2}\right)\right) \text { holds }
\end{aligned}
$$

iff there is an edge labeled by 0 (respectively, by 1 ) from $v_{1}$ to $v_{2}$.

$$
v_{1} \prec v_{2} \text { iff } v_{1}=\left\langle i_{1}, j_{1}\right\rangle \text { and } v_{2}=\left\langle i_{2}, j_{2}\right\rangle \text { and } j_{1}<j_{2} .
$$

The next lemma shows that the set of memoryless winning strategies is definable in $M_{\mathcal{A}, \mathbf{P}}$.

Lemma 6.3 (The set of memoryless winning strategies is definable in $M_{\mathcal{A}, \mathbf{P}}$ ). Let $\mathcal{A}$ be a parity automaton.

(1) There is a monadic second-order formula win $S t_{G_{\mathcal{A}}}^{I}(X)$ such that $M_{\mathcal{A}, \mathbf{P}} \models \operatorname{win} t_{G_{\mathcal{A}}}^{I}(U)$ iff $U$ corresponds to a memoryless winning strategy for Player $I$.

(2) There is a monadic second-order formula win $S t_{G_{\mathcal{A}}}^{I I}(X)$ such that $M_{\mathcal{A}, \mathbf{P}} \models$ win $S t_{G_{\mathcal{A}}}^{I I}(U)$ iff $U$ corresponds to a memoryless winning strategy for Player II.

(3) Moreover, win $S t_{G_{\mathcal{A}}}^{I}(X)$ and win $S t_{G_{\mathcal{A}}}^{I I}(X)$ are computable from $\mathcal{A}$.

Proof. We will formalize that "player I wins all the plays consistent with a memoryless strategy $X "$.

A play is an infinite path that starts from the initial node. Note that the arena $G_{\mathcal{A}, \mathbf{P}}$ is an acyclic graph. Hence, we can formalize that " $Z$ is the set of nodes of an infinite path that starts from a node $v "$ as a formula $\operatorname{Play}(v, Z)$ which is the conjunction of the following formulas:

(1) For every node $u$ of $Z$ there is a unique node $u^{\prime} \in Z$ such that an edge from $u$ enters $u^{\prime}$.

(2) For every node $u \neq v$ of $Z$ there is a unique node $u^{\prime} \in Z$ such that an edge from $u^{\prime}$ enters $u$; there is no edge that enters from a node of $Z$ into $v$.

(3) $v$ is in $Z$.

(4) For every partition of $Z$ into two non-empty set $Z_{1}$ and $Z_{2}$ there is an edge between a node in $Z_{1}$ and a node in $Z_{2}$.

The assertion " $u$ is a Player I node" is formalized by the formula $\operatorname{Pos}_{1}(u)$, defined as $\bigvee_{q \in \mathcal{Q}} R_{q}(u)$. Next, we formalize that " $Z$ is the set of nodes of a play consistent with a strategy $U$ of Player I" by the formula $\operatorname{Consis}(U, Z)$ which is the conjunction of the following formulas:

(1) " $U$ is a subset of Player I nodes" is formalized by

$$
\forall u\left(u \in U \rightarrow \operatorname{Pos}_{1}(u)\right)
$$

(2) " $Z$ is the set of nodes of a play from the initial node":

$$
\exists v(\operatorname{Init}(v) \wedge \operatorname{Play}(v, Z))
$$

(3) " $Z$ is consistent with $U$ "

$$
\forall z z^{\prime} \in Z\left(\operatorname{Pos}_{1}(z) \wedge\left(E_{1}\left(z, z^{\prime}\right) \vee E_{1}\left(z . z^{\prime}\right)\right)\right) \rightarrow\left(E_{1}\left(z, z^{\prime}\right) \leftrightarrow z \in U\right)
$$


Assume that the coloring function of $\mathcal{A}$ assigns to the states numbers in the set $\{0,1 \ldots, m\}$, and let $Q_{i}(i=0, \ldots m)$ be the set of states of $\mathcal{A}$ which are colored by $i$. Color $i$ appears infinitely often in a play with the set of nodes $Z$ if $\inf _{i}(Z)$ defined as $\forall z \exists z^{\prime} \in Z\left(z \prec z^{\prime} \wedge\left(\vee_{q \in Q_{i}} R_{q}\left(z^{\prime}\right)\right)\right)$ holds. Hence, the formula $\operatorname{Even}(Z)$ defined as

$$
\bigvee_{k \leq m / 2}\left(i n f_{2 k}(Z) \wedge \bigwedge_{j<2 k} \neg i n f_{2 k}(Z)\right)
$$

holds for a play $Z$ iff the minimal color that appears infinitely often in $Z$ is even.

Finally, win $S t_{G_{\mathcal{A}}}^{I}(X)$ can be defined as $\forall Z(\operatorname{Consis}(X, Z) \rightarrow \neg \operatorname{Even}(Z))$.

Note that our construction of $\operatorname{win} S t_{G_{\mathcal{A}}}^{I}(X)$ from $\mathcal{A}$ is algorithmic.

The formula win $S t_{G_{\mathcal{A}}}^{I I}(X)$ is defined from $\mathcal{A}$ similarly.

Next, we will show that the operator $F_{U}:\{0,1\}^{\omega} \rightarrow\{0,1\}^{\omega}$ induced by a memoryless strategy $U$ of one of the players is definable in $M_{\mathcal{A}, \mathbf{P}}$. The operator $F_{U}$ maps $\omega$-strings to $\omega$ strings. Therefore, we should agree how the $\omega$-strings are encoded by subset of $M_{\mathcal{A}, \mathbf{P}}$.

Note that for $q \in \mathcal{Q}$ the set of elements in $R_{q}$ ordered by $\prec$ is isomorphic to $\langle$ Nat,$<\rangle$. Accordingly, we can represent the $\omega$-strings by the subsets of $R_{\text {qinit }}$, and in the next lemma the operators from $\{0,1\}^{\omega}$ to $\{0,1\}^{\omega}$ are identified with corresponding functions from the set of subset of $R_{q_{\text {init }}}$ to the set of subset of $R_{q_{\text {init }}}$.

Lemma 6.4 (Definability of the operator induced by a memoryless strategy). Let $\mathcal{A}$ be a parity automaton.

(1) There is a monadic second-order formula $\psi_{I}(X, Y, U)$ such that $M_{\mathcal{A}, \mathbf{P}} \models \psi_{I}(\mathbf{X}, \mathbf{Y}, \mathbf{U})$ iff $\mathbf{U}$ is a memoryless strategy for Player $I$ in $G_{\mathcal{A}, \mathbf{P}}, \mathbf{X}, \mathbf{Y} \subseteq R_{q_{\text {init }}}$ and $\mathbf{X}=F_{\mathbf{U}}(\mathbf{Y})$, where $F_{\mathbf{U}}$ is the operator induced by $\mathbf{U}$.

(2) There is a monadic second-order formula $\psi_{I I}(X, Y, U)$ such that $M_{\mathcal{A}, \mathbf{P}} \models \psi_{I I}(\mathbf{X}, \mathbf{Y}, \mathbf{U})$ iff $\mathbf{U}$ is a memoryless strategy for Player II in $G_{\mathcal{A}, \mathbf{P}}, \mathbf{X}, \mathbf{Y} \subseteq R_{q_{\text {init }}}$ and $\mathbf{Y}=F_{\mathbf{U}}(\mathbf{X})$, where $F_{\mathbf{U}}$ is the operator induced by $\mathbf{U}$.

(3) Moreover, $\psi_{I}$ and $\psi_{I I}$ are computable from $\mathcal{A}$.

Proof. We just formalize in the monadic-second order logic the construction of $F_{U}$ given in Sect. 6.2,

Let $\operatorname{Consis}(U, Z)$ be the formula from the proof of Lemma 6.3 which expresses " $Z$ is the set of nodes of a play consistent with a strategy $U$ of Player I". We need to say that $X$ (respectively, $Y$ ) is the sequence of edges 1 chosen by Player I (respectively, by Player II) in the play $Z$. It can be formalized by formula moves $(X, Y, Z)$ which is the conjunction of

(1) $X \subseteq R_{q_{\text {init }}} \wedge Y \subseteq R_{q_{\text {init }}}-X$ and $Y$ "encodes" $\omega$ strings over $\{0,1\}$.

(2) We can formalize that $X$ is the sequence of edges chosen by Player I as follows. Let $u \in Z$ be a node of Player II. Then, $u$ is $\langle q, 1, j\rangle$ iff $\left\langle q_{\text {init }}, j\right\rangle$ is in $X$ :

$$
\forall u \in Z \forall v \in X\left(\operatorname{Pos}_{I I}(u) \wedge \neg(u \prec v) \wedge \neg(v \prec u)\right) \rightarrow\left(v \in X \leftrightarrow \bigvee_{q \in \mathcal{Q}} u \in R_{q, 1}\right)
$$

(3) Similarly, we can formalize that $Y$ is the sequence of edges chosen by Player II as follows. Let $u=\langle q, a, j\rangle \in Z$ and $u^{\prime}=\left\langle q^{\prime}, j+1\right\rangle \in Z$ and let $c$ be 1 (respectively, $c$ be 0 ) if $j \in \mathbf{P}$ (respectively, $j \notin \mathbf{P}$ ) and let $b$ be 1 (respectively, $b$ be 0 ) if $\left\langle q_{\text {init }}, j\right\rangle \in Y$ (respectively, $\left.\left\langle q_{\text {init }}, j\right\rangle \notin Y\right)$. Then, $q^{\prime}=\delta_{\mathcal{A}}(q,\langle a, b, c\rangle)$.

\footnotetext{
${ }^{1}$ Strictly speaking, the sequence of labels of the edges.
} 
Note that for each memoryless strategy $U$ of Player I, we have

$$
\forall Y \subseteq R_{q_{\text {init }}} \exists ! Z \exists ! X \operatorname{Consis}(U, Z) \wedge \operatorname{moves}(X, Y, Z)
$$

. Finally, $\psi_{I}(X, Y, U)$ can be defined as $\exists Z \operatorname{Consis}(U, Z) \wedge \operatorname{moves}(X, Y, Z)$.

$\psi_{I I}(X, Y, U)$ is defined in a similar way.

6.4. Interpretation of $M_{\mathcal{A}, \mathbf{P}}$ in the structure $\langle N a t,<\mathbf{P}\rangle$. Every set $S$ of nodes in $M_{\mathcal{A}, \mathbf{P}}$ corresponds to the tuple $\left\langle\ldots, W_{i}, \ldots\right\rangle$ where $\left.i \in \mathcal{Q} \cup \mathcal{Q} \times\{0,1\}\right)$ of subsets of Nat, such that $\langle q, m\rangle \in S$ iff $m \in W_{q}$ and $\langle q, a, m\rangle \in S$ iff $m \in W_{\langle q, a\rangle}$.

The proof of the following lemma shows that there is an interpretation of the structure $M_{\mathcal{A}, \mathbf{P}}$ in the structure $\langle$ Nat,$<\mathbf{P}\rangle$.

Lemma 6.5. For every formula $\psi\left(X^{1}, \ldots X^{k}\right)$ in the second order monadic logic over the signature $\tau_{\mathcal{A}}$ with free monadic variables $X^{1}, \ldots, X^{k}$ there is a formula $\varphi\left(Y, \overrightarrow{Z^{1}} \ldots, \overrightarrow{Z^{k}}\right)$, where $\overrightarrow{Z^{j}}$ is a tuple of monadic variables $\left\{Z_{i}^{j}: i \in \mathcal{Q} \cup \mathcal{Q} \times\{0,1\}\right\}$, such that for every $\mathbf{P} \subseteq$ Nat and a tuple $\left\langle\ldots, W_{i}^{j}, \ldots\right\rangle$ where $j \in\{1, \ldots, k\}$ and $i \in \mathcal{Q} \cup \mathcal{Q} \times\{0,1\}$ ) of subsets of Nat the following equivalence holds:

$$
\langle N a t,<\rangle \models \varphi\left(\mathbf{P}, \ldots, W_{i}^{j}, \ldots\right) \text {, iff } M_{\mathcal{A}, \mathbf{P}} \models \psi\left(S^{1}, \ldots, S^{k}\right)
$$

where $S^{j}$ is the subset of nodes in $G_{\mathcal{A}, \mathbf{P}}$, which corresponds to $\left\langle\ldots, W_{i}^{j}, \ldots\right\rangle$. Moreover, there is an algorithms that computes $\varphi$ from $\psi$.

Proof. The proof proceeds by the structural induction. It is more convenient to consider the first-order version of the monadic second-order logic (see, Subsection 4.3).

Basis - Atomic formulas.

- $\operatorname{Sing}(X)$ is translated as

$$
\bigvee_{i \in \mathcal{Q} \cup \mathcal{Q} \times\{0,1\}} \operatorname{Sing}\left(Z_{i}\right) \wedge \bigwedge_{i \neq i^{\prime} \in \mathcal{Q} \cup \mathcal{Q} \times\{0,1\}}\left(\operatorname{Sing}\left(Z_{i}\right) \rightarrow \operatorname{Empty}\left(Z_{i^{\prime}}\right)\right),
$$

where $\operatorname{Empty}(W)$ is a shorthand for $\forall W^{\prime}\left(W \subseteq W^{\prime}\right)$.

- $X^{1} \subseteq X^{2}$ is translated as

$$
\bigwedge_{i \in \mathcal{Q} \cup \mathcal{Q} \times\{0,1\}} Z_{i}^{1} \subseteq Z_{i}^{2}
$$

- $X^{1} \prec X^{2}$ is translated as the conjunction of the following:

(1) one of the $Z_{i}^{1}(i \in \mathcal{Q} \cup \mathcal{Q} \times\{0,1\})$ is singleton and all the others are empty.

(2) one of the $Z_{i}^{2}(i \in \mathcal{Q} \cup \mathcal{Q} \times\{0,1\})$ is singleton and all the others are empty.

(3) if $Z_{i_{1}}^{1}$ and $Z_{i_{2}}^{2}$ are singletons, then $Z_{i_{1}}^{1}<Z_{i_{2}}^{2}$, i.e., the (unique) natural number which is in $Z_{i_{1}}^{1}$ is less than the natural number in $Z_{i_{2}}^{2}$.

- Other relations in $\tau_{\mathcal{A}}$ are translated similarly just by the straightforward formalization of our definition of the game arena, e.g., $E_{0}\left(X^{1}, X^{2}\right)$ is translated as the conjunction of the following:

(1) one of the $Z_{i}^{1}(i \in \mathcal{Q} \cup \mathcal{Q} \times\{0,1\})$ is singleton and all others are empty.

(2) one of the $Z_{i}^{2}(i \in \mathcal{Q} \cup \mathcal{Q} \times\{0,1\})$ is singleton and all others are empty.

(3) if $Z_{q}^{1}$ (for some $q \in \mathcal{Q}$ ) is singleton, then $Z_{\langle q, 0\rangle}^{2}$ is singleton and both of them contain the same natural number. 
(4) if $Z_{\langle q, a\rangle}^{1}$ (for some $q \in \mathcal{Q}$ and $a \in\{0,1$,$\} ) is singleton, and the unique number n$ from $Z_{\langle q, a\rangle}^{1}$ is in $\mathbf{P}$ (i.e., $\left.Z_{\langle q, a\rangle}^{1} \subseteq \mathbf{P}\right)$ and $q^{\prime}$ is be $\delta_{\mathcal{A}}(q,\langle a, 0,1\rangle)$, then $Z_{q^{\prime}}^{2}$ is singleton and the unique element of $Z_{q^{\prime}}^{2}$ is the successor of the unique element of $Z_{\langle q, a\rangle}^{1}$.

(5) if $Z_{\langle q, a\rangle}^{1}$ (for some $q \in \mathcal{Q}$ and $a \in\{0,1$,$\} ) is singleton, and the unique number n$ from $Z_{\langle q, a\rangle}^{1}$ is not in $\mathbf{P}$ (i.e., $\left.\neg\left(Z_{\langle q, a\rangle}^{1} \subseteq \mathbf{P}\right)\right)$ and $q^{\prime}$ is $\delta_{\mathcal{A}}(q,\langle a, 0,0\rangle)$, then $Z_{q^{\prime}}^{2}$ is singleton, and the unique element of $Z_{q^{\prime}}^{2}$ is the successor of the unique element of $Z_{\langle q, a\rangle}^{1}$.

Inductive step. If $\varphi_{j}$ is the translation of $\psi_{j}$ for $j=1,2$, then $\psi_{1} \wedge \psi_{2}$ is translated as $\varphi_{1} \wedge \varphi_{2}$ and $\neg \psi_{1}$ is translated as $\neg \varphi_{1}$.

Finally, if $\varphi\left(Y, \overrightarrow{Z^{1}} \ldots, \overrightarrow{Z^{k}}\right)$ is the translation of $\psi\left(X^{1}, \ldots X^{k}\right)$, then $\exists X^{1} \psi$ is translated as $\exists \overrightarrow{Z^{1}} \varphi$.

The next two lemmas use the interpretation of $M_{\mathcal{A}, \mathbf{P}}$ in $\langle N a t,<\mathbf{P}\rangle$ to show $M L O$ definability in $\langle N a t,<\mathbf{P}\rangle$ of the set of memoryless winning strategies and the operator induced by a memoryless strategy.

Lemma 6.6 (The set of memoryless winning strategies is definable in $\langle$ Nat, $<\mathbf{P}\rangle$ ).

Let $\mathcal{A}=\left\langle\mathcal{Q}, \Sigma, \delta_{\mathcal{A}}, q_{\text {init }}\right.$, col $\rangle$, be a deterministic parity automaton over the alphabet $\Sigma=$ $\{0,1\} \times\{0,1\} \times\{0,1\}$.

(1) There is an $M L O$ formula $W_{i n S t}^{I}\left(Z_{1}, \ldots, Z_{|\mathcal{Q}|}, Z\right)$ such that for every $\mathbf{P} \subseteq$ Nat and $W_{1}, \ldots, W_{|\mathcal{Q}|} \subseteq$ Nat:

$$
N a t \models W i n S t t_{\mathcal{A}}^{I}\left(W_{1}, \ldots, W_{|\mathcal{Q}|}, \mathbf{P}\right)
$$

iff the corresponding subset $U \subseteq \mathcal{Q} \times N$ at defines a memoryless winning strategy for Player I in $G_{\mathcal{A}, \mathbf{P}}$.

(2) There is an $M L O$ formula $\operatorname{WinSt}_{\mathcal{A}}^{I I}\left(Z_{1}, \ldots, Z_{|\mathcal{Q}|}, Z_{1}^{\prime}, \ldots, Z_{|\mathcal{Q}|}^{\prime}, Z\right)$ such that for every $\mathbf{P} \subseteq$ Nat and $W_{1}, \ldots, W_{|\mathcal{Q}|}, W_{1}^{\prime}, \ldots, W_{|\mathcal{Q}|}^{\prime} \subseteq$ Nat:

$$
N a t \models \operatorname{WinSt}_{\mathcal{A}}^{I I}\left(W_{1}, \ldots, W_{|\mathcal{Q}|}, W_{1}^{\prime}, \ldots, W_{|\mathcal{Q}|}^{\prime}, \mathbf{P}\right)
$$

iff the corresponding subset $U \subseteq \mathcal{Q} \times\{0,1\} \times$ Nat defines a memoryless winning strategy for Player II in $G_{\mathcal{A}, \mathbf{P}}$.

(3) Moreover, there is an algorithm that computes formulas $\operatorname{WinSt}_{\mathcal{A}}^{I}$ and $\operatorname{WinSt}_{\mathcal{A}}^{I I}$ from $\mathcal{A}$.

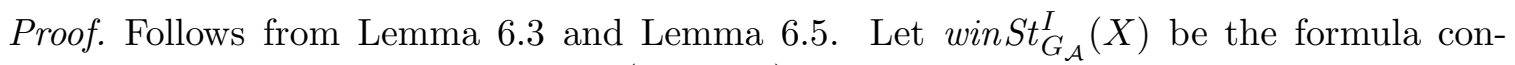
structed in Lemma 6.3 which defines (in $M_{\mathcal{A}, \mathbf{P}}$ ) the set of memoryless winning strategies of the first player. Let $\psi\left(Y, \vec{Z}^{1}\right)$ be its translation, as in Lemma 6.5, where $\overrightarrow{Z^{1}}$ is the tuple of variables indexed by $i \in \mathcal{Q} \cup \mathcal{Q} \times\{0,1\}$. Note that since $\overrightarrow{Z^{1}}$ defines (in $M_{\mathcal{A}, \mathbf{P}}$ ) a strategy of Player I, $Z_{\langle q, a\rangle}$ for $q \in \mathcal{Q}$ and $a \in\{0,1\}$ should be interpreted as the empty set. The formula $\operatorname{WinSt}_{\mathcal{A}}^{I}$ is obtained from $\psi$ by replacing $Z_{\langle q, a\rangle}$ for $q \in \mathcal{Q}$ and $a \in\{0,1\}$ by the empty set and replacing $Y$ by $Z$.

$\operatorname{WinSt}_{\mathcal{A}}^{I I}$ is defined in a similar way.

From Lemma 6.4 and Lemma 6.5, by the same arguments, we can derive the following Lemma:

Lemma 6.7 (The operator induced by a memoryless strategy is definable in $\langle$ Nat, $<\mathbf{P}\rangle$ ). Let $\mathcal{A}=\left\langle\mathcal{Q}, \Sigma, \delta_{\mathcal{A}}, q_{\text {init }}\right.$, col $\rangle$, be a deterministic parity automaton over the alphabet $\Sigma=$ $\{0,1\} \times\{0,1\} \times\{0,1\}$. 
(1) There is an $M L O$ formula $o p_{\mathcal{A}}^{I}\left(X, Y, Z_{1}, \ldots, Z_{|\mathcal{Q}|}, Z\right)$ which has the following property: Let $\mathbf{P} \subseteq N a t$ and $W_{1}, \ldots, W_{|\mathcal{Q}|} \subseteq N a$, and let $F_{U}$ be the C-operator, induced by Player I memoryless strategy $U \subseteq \mathcal{Q} \times$ Nat which corresponds to $\left\langle W_{1}, \ldots, W_{|\mathcal{Q}|}\right\rangle$. Then, $N a t \models o p_{\mathcal{A}}^{I}\left(X, Y, W_{1}, \ldots, W_{|\mathcal{Q}|}, \mathbf{P}\right)$ iff $X=F_{U}(Y)$.

(2) There is an $M L O$ formula $o_{\mathcal{A}}^{I I} \mathcal{A}\left(X, Y, Z_{1}, \ldots, Z_{|\mathcal{Q}|}, Z_{1}^{\prime}, \ldots, Z_{|\mathcal{Q}|}^{\prime}, Z\right)$ which has the following property:

Let $\mathbf{P} \subseteq N a t$ and $W_{1}, \ldots, W_{|\mathcal{Q}|}, W_{1}^{\prime}, \ldots, W_{|\mathcal{Q}|}^{\prime} \subseteq N a t$ and let $F_{U}$ be the $C$ operator, induced by Player II memoryless strategy $U \subseteq \mathcal{Q} \times\{0,1\} \times N a t$ which corresponds to $\left\langle W_{1}, \ldots, W_{|\mathcal{Q}|}, W_{1}^{\prime}, \ldots, W_{|\mathcal{Q}|}^{\prime}\right\rangle$. Then,

$$
N a t \models o p_{\mathcal{A}}^{I I}\left(X, Y, W_{1}, \ldots, W_{|\mathcal{Q}|}, W_{1}^{\prime}, \ldots, W_{|\mathcal{Q}|}^{\prime}, \mathbf{P}\right) \text { iff } Y=F_{U}(X) .
$$

(3) Moreover, there is an algorithm that computes formulas op ${ }_{\mathcal{A}}^{I}$ and $o p_{\mathcal{A}}^{I I}$ from $\mathcal{A}$.

\section{Proof of Theorem 2.3}

We are now almost ready to prove Theorem 2.3. We will show an algorithm that, given a formula $\varphi(X, Y, P)$, constructs

(1) a sentence $\operatorname{WIN}_{\varphi}^{I I}(P)$ and

(2) formulas $S t_{\varphi}^{I}(X, Y, P)$ and $S t_{\varphi}^{I I}(X, Y, P)$

such that for every structure $M=\langle N a t,<, \mathbf{P}\rangle$, Player II has a winning strategy in the games $\mathcal{G}_{\varphi}^{M}$ iff $M \models W I N_{\varphi}^{I I}$. If Player II (respectively, Player I) has a winning strategy, then $S t_{\varphi}^{I I}(X, Y, P)$ (respectively, $\left.S t_{\varphi}^{I}(X, Y, P)\right)$ defines his winning strategy.

Let $\varphi(X, Y, P)$ be a formula. We are going to construct $\operatorname{WIN}_{\varphi}^{I I}(P)$, as follows. First, let $\varphi^{\prime}(X, Y, Z)$ be a formula obtained from $\varphi$ by replacing all the occurrences of $P$ by a fresh variable $Z$.

Let $\mathcal{A}=\left\langle\mathcal{Q}, \Sigma, \delta_{\mathcal{A}}, q_{\text {init }}, \operatorname{col}\right\rangle$ be a deterministic parity automaton over the alphabet $\Sigma=\{0,1\} \times\{0,1\} \times\{0,1\}$, which is equivalent to $\varphi^{\prime}$. Let $\operatorname{WinSt}_{\mathcal{A}}^{I}\left(Z_{1}, \ldots, Z_{|\mathcal{Q}|}, Z\right)$ be constructed from $\mathcal{A}$, as in Lemma 6.6. Player I has a winning strategy iff

$$
M \models \exists Z_{1} \ldots \exists Z_{|\mathcal{Q}|} \operatorname{WinSt}_{\mathcal{A}}^{I}\left(Z_{1}, \ldots, Z_{|\mathcal{Q}|}, P\right) .
$$

Finally, $\operatorname{WIN}_{\varphi}^{I I}(P)$ can be defined as $\neg \exists Z_{1} \ldots \exists Z_{|\mathcal{Q}|} \operatorname{WinSt}_{\mathcal{A}}^{I}\left(Z_{1}, \ldots, Z_{|\mathcal{Q}|}, P\right)$.

The correctness of the construction follows from Lemma 6.6 and Lemma 6.2.

In order to construct $S t_{\varphi}^{I}(X, Y, P), S t_{\varphi}^{I I}(X, Y, P)$, we need the following definition and Theorem.

Definition 7.1 (Selection). Let $\varphi(\bar{Y}), \psi(\bar{Y})$ be formulas and $C$ a class of structures. We say that $\psi$ selects (or, is a selector for) $\varphi$ over $C$ iff for every $M \in C$ :

(1) $M \models \exists \leq 1 \bar{Y} \psi(\bar{Y})$,

(2) $M \models \forall \bar{Y}(\psi(\bar{Y}) \rightarrow \varphi(\bar{Y}))$, and

(3) $M \models \exists \bar{Y} \varphi(\bar{Y}) \rightarrow \exists \bar{Y} \psi(\bar{Y})$.

Here, $\bar{Y}$ is a tuple of distinct variables and " $\exists \leq 1 \bar{Y} \ldots$. stands for "there exists at most one...". The definition can be rephrased as $\psi$ is a selector of $\varphi$ over $C$ iff for each $M \in C$, if $\varphi$ is satisfiable in $M$, then it is satisfiable by the (unique) tuple defined by $\psi$.

We say that $C$ has the selection property iff every formula $\varphi$ has a selector $\psi$ over $C$. 
Theorem 7.2. The class of labelled $\omega$-chains has the selection property. Moreover, there is an algorithm which constructs for every $\varphi$ a formula $\psi$ which selects $\varphi$ over the class of labelled $\omega$-chains.

Theorem 7.2 was proved in Rab07]. Its version without the "Moreover" clause was stated without proof in [LS98.

Now, we are ready to define $S t_{\varphi}^{I}(X, Y, P)$ and $S t_{\varphi}^{I I}(X, Y, P)$.

Let $\mathcal{A}=\left\langle\mathcal{Q}, \Sigma, \delta_{\mathcal{A}}, q_{\text {init }}, \operatorname{col}\right\rangle$ and $\operatorname{WinSt}_{\mathcal{A}}^{I}\left(Z_{1}, \ldots, Z_{|\mathcal{Q}|}, Z\right)$ be as in the construction of $\operatorname{WIN}_{\varphi}^{I I}(P)$ above.

Construct $\alpha\left(Z_{1}, \ldots, Z_{|\mathcal{Q}|}, P\right)$ as a selector for $\operatorname{WinSt}_{\mathcal{A}}^{I}\left(Z_{1}, \ldots, Z_{|\mathcal{Q}|}, P\right)$ over the class $\{\langle N a t,<, \mathbf{P}\rangle: \mathbf{P} \subseteq N a t\}$ of structures. If $\operatorname{WinSt}_{\mathcal{A}}^{I}\left(Z_{1}, \ldots, Z_{|\mathcal{Q}|}, P\right)$ is satisfiable in $M$, then every tuple which satisfies it corresponds to a memoryless winning strategy of Player I in the parity game $G_{\mathcal{A}, \mathbf{P}}$. In particular, the tuple defined by $\alpha$ describes a memoryless winning strategy of Player I in the parity game $G_{\mathcal{A}, \mathbf{P}}$. Let $o p_{\mathcal{A}}^{I}$ be as in Lemma 6.6. Then, $\exists Z_{1} \ldots \exists Z_{|\mathcal{Q}|}\left(\alpha\left(Z_{1}, \ldots, Z_{|\mathcal{Q}|}, P\right) \wedge o p_{\mathcal{A}}^{I}\left(X, Y, Z_{1}, \ldots, Z_{|\mathcal{Q}|}\right)\right.$ defines in $M$ a SC-operator $F$, induced by a memoryless winning strategy of Player I in the parity game $G_{\mathcal{A}, \mathbf{P}}$.

Hence, by Lemma 6.2, $\neg R_{\mathcal{A}}(F(Y), Y, \mathbf{P})$ holds for every $Y$. Therefore, by the definitions of $\mathcal{A}$ and $R_{\mathcal{A}}$, we have

$$
\langle N a t,<\rangle \models \forall Y \neg \psi((F(Y), Y, \mathbf{P}) .
$$

Hence $F$, defined by $\exists Z_{1} \ldots \exists Z_{|\mathcal{Q}|}\left(\alpha \wedge o p_{\mathcal{A}}^{I}\left(X, Y, Z_{1}, \ldots, Z_{|\mathcal{Q}|}\right)\right.$, is a winning strategy for Player I in $\mathcal{G}_{\varphi}^{M}$.

The formula $S t_{\varphi}^{I I}(X, Y, P)$ which defines a winning strategy for Player II is constructed similarly.

\section{Conclusion and Related Work}

We investigated the Church synthesis problem with parameters. We provided the necessary and sufficient conditions for the computability of Synthesis problems 1-3.

The conditions of Theorem 5.1 and Theorem 5.2 are sufficient, but are not necessary for the computability of Synthesis problems 4-5. For example, let Fac $=\{n !: n \in$ Nat $\}$ be the set of factorial numbers. We can show that Problems 4-5 are computable for this predicate Fac Rab06.

It is an open question whether the decidability of $\langle N a t,\langle, \mathbf{P}\rangle$ is a sufficient condition for the computability of Synthesis problems 4-5.

We proved that the definability and synthesis parts of the Büchi and Landweber theorem hold for all expansions of $\omega$ by unary predicates.

Büchi proved that the $M L O$-theory of any countable ordinal is decidable. After stating their main theorem, Büchi and Landweber write:

"We hope to present elsewhere a corresponding extension of [our main theorem] from $\omega$ to any countable ordinal."

However, despite the fundamental role of the Church problem, no such extension is even mentioned in a later book by Büchi and Siefkes [BS73], which summarizes the theory of finite automata and $M L O$ over words of countable length.

In Rab06a, we proved that the determinacy and decidability parts of the Büchi and Landweber theorem hold for all countable ordinals; however, its definability and synthesis parts hold for an ordinal $\alpha$ iff $\alpha<\omega^{\omega}$ [RS06]. 
In [RT07], the Church Problem for fragments of $M L O$ was considered. First-order $M L O$ formulas are $M L O$ formulas without the second-order quantifiers. In [RT06], it was proved that if $\psi(X, Y)$ is a first-order $M L O$ formula, then one of the players has a first-order definable winning strategy in $\mathcal{G}_{\psi}^{\omega}$. Similar results were obtained for several interesting fragments of $M L O$. However, it is an open question whether these results hold when parameters are added.

Kupferman and Vardi [KV97] considered the synthesis problem with incomplete information for the specifications described by temporal logics LTL and CTL*. Their main results deal with the complexity of this synthesis problem. The decidability of the synthesis problem with incomplete information for LTL (respectively, for CTL*) can be easily derived from the Büchi-Landweber (respectively, Rabin) theorem. It seems that there are no interesting connections between the synthesis problems with incomplete information and the synthesis problems with parameters considered here.

In [RT98] a program for the relativization of finite automata theory was proposed. Our results can be seen as the first step in this direction. This step corresponds to the case where oracles are $\mathrm{C}$-operators without inputs.

In the rest of this section we comment on Rabin's proof of the Church synthesis Problem, discuss possibilities of extending it to the Church synthesis Problem with parameters, and state some open questions.

Rabin [Rab72] provided an alternative proof for computability of the Church synthesis problem. This proof uses an automata on infinite trees as a natural tool for treating the synthesis problem. A C-operator $F:\{0,1\}^{\omega} \rightarrow\{0,1\}^{\omega}$ can be represented by a labelled infinite full binary tree $\left\langle T_{2},<, \mathbf{S}\right\rangle$, where $\mathbf{S}$ is a subset of the tree nodes. Namely, the branches of the tree represent $X \in\{0,1\}^{\omega}$ and the sequence of values assigned by $\mathbf{S}$ to the nodes along the branch $X$ represents $F(X)=Y \in\{0,1\}^{\omega}$. Also, the fact that $\mathbf{S}$ represents a C-operator $F$ which uniformizes $\varphi(X, Y)$ can be expressed by an $M L O$ formula $\psi(Z)$ (computable from $\varphi(X, Y)): T_{2} \models \psi(\mathbf{S})$ iff $N a t \models \forall \varphi\left(X, F_{\mathbf{S}}(X)\right)$, where $F_{\mathbf{S}}$ is the C-operator that corresponds to $\mathbf{S}$. Hence, the question whether there exists a $\mathrm{C}$-operator which uniformizes $\varphi$ is reduced to the problem whether $T_{2} \models \exists Z \psi(Z)$. Now, the Rabin basis theorem states that if $T_{2} \models \exists Z \psi(Z)$ then there is a regular subset $\mathbf{S} \subseteq T_{2}$ such that $T_{2} \models \psi(\mathbf{S})$. The C-operator which corresponds to a regular set $\mathbf{S}$ is computable by a finite state automaton. Hence, the Büchi and Landweber theorem is obtained as a consequence of the decidability of the monadic logic of order of the full binary tree and the basis theorem.

One could try to apply the Rabin method to the Church synthesis problem with parameters. The reduction which is similar to Rabin's reduction shows that for every $\varphi(X, Y, P)$ there is a sentence $\operatorname{WIN}_{\varphi}^{I I}(Q)$ such that for every structure $M=\langle$ Nat, $<, \mathbf{P}\rangle$ Player II wins the games $\mathcal{G}_{\varphi}^{M}$ iff

$$
\left\langle T_{2},<, \mathbf{Q}\right\rangle \models \operatorname{WIN}_{\varphi}^{I I}(Q),
$$

where a node is in $\mathbf{Q}$ if its distance from the root is in $\mathbf{P}$. Moreover, $\operatorname{WIN}_{\varphi}^{I I}(Q)$ is computable from $\varphi$ (cf. Theorem 2.3).

Hence, the decidability of Problem $1^{\prime}$ for $\mathbf{P} \subseteq$ Nat (recall that this is Problem 1 without its constructive part, see Sect. 3) is reduced to the decidability of the monadic theory of the labelled full binary tree $\left\langle T_{2},<, \mathbf{Q}\right\rangle$, where a node is in $\mathbf{Q}$, if its distance from the root is in $\mathbf{P}$. The decidability of the latter problem can be reduced by Shelah-Stupp Muchnick Theorem Shel75, Wal02, Th03 to the decidability of $\langle$ Nat, $<, \mathbf{P}\rangle$. Now, in order to establish computability of problems 1-3, one can try to prove the basis theorem for $\left\langle T_{2},<, \mathbf{Q}\right\rangle$. 
Unfortunately, arguments similar to the proof of Theorem 5.1 show that for $\mathbf{P}, \mathbf{Q}$, as above, the following are equivalent:

(1) $\mathbf{P}$ is ultimately periodic.

(2) For every $\psi(Z, Q)$ such that $T_{2} \models \exists Z \psi(Z, \mathbf{Q})$ there is a finite state operator $F(Y, U)$ such that the set which corresponds to the C-operator $\lambda X F(X, \mathbf{P})$ satisfies $\psi(Z, \mathbf{Q})$.

In case selector over $\left\langle T_{2},<, \mathbf{Q}\right\rangle$ is computable, it would be easy to derive the computability of Problems 1-3. However, even the following is open

Open Question - Selectoion property for an expansion of $T_{2}$ by unary predicate.

Is it true that for every $\mathbf{Q} \subseteq T_{2}$ every formula $\varphi(Y, Q)$ has a selector over

$\left\langle T_{2},<, \mathbf{Q}\right\rangle$, i.e., there is $\psi(Y, Q)$ such that $(1)\left\langle T_{2},<. \mathbf{Q}\right\rangle \models \exists^{\leq 1} Y \psi(Y)$, (2)

$\left\langle T_{2},<, \mathbf{Q}\right\rangle \models \forall Y(\psi(Y) \rightarrow \varphi(Y))$, and $(3)\left\langle T_{2},<\mathbf{Q}\right\rangle \models \exists Y \varphi(Y) \rightarrow \exists \bar{Y} \psi(Y)$.

Note that this question asks whether every expansion of $T_{2}$ has the selection property and is different from the Rabin's uniformization problem over $T_{2}$, which asks whether every formula has a selector over the class of all the expansions of $T_{2}$ by unary predicates. The negative answer to the Rabin uniformization problem was obtained by Gurevich and Shelah GS83 who proved that a formula $\varphi(Y, Q)$ which express that "if $Q$ is not empty than $Y$ is a singleton set which is a subset of $Q$ " has no selector over the class of all the expansions of $T_{2}$.

We believe that if the answer to the above mentioned question is positive, then its proof is non-trivial.

Our proof of Theorem 1.2 implies that for $\mathbf{P} \subseteq$ Nat and $\mathbf{Q} \subseteq T_{2}$, where a node is in $\mathbf{Q}$ if its distance from the root is in $\mathbf{P}$, the following are equivalent:

(1) The monadic theory of $\langle N a t,<, \mathbf{P}\rangle$ is decidable,

(2) For every $\psi(Z, Q)$ such that $T_{2} \models \exists Z \psi(Z, \mathbf{Q})$ there is a recursive $\mathbf{S} \subset T_{2}$ such that $T_{2} \models \psi(\mathbf{S}, \mathbf{Q})$.

However, we do not know the answer to the following question:

Open Question: Are the following assertions equivalent?

(1) The monadic theory of $\left\langle T_{2},<\mathbf{S}\right\rangle$ is decidable.

(2) For every $\psi(Z, U)$ such that $T_{2} \models \exists Z \psi(Z, \mathbf{S})$ there is a recursive set $\mathbf{Q} \subset T_{2}$ such that $T_{2} \models \psi(\mathbf{Q}, \mathbf{S})$.

\section{ACKNOWLEDGMENTS}

I am grateful to the anonymous referees for their suggestions.

\section{REFERENCES}

[Bu60] J. R. Büchi. On a decision method in restricted second order arithmetic In Proc. International Congress on Logic, Methodology and Philosophy of Science, E. Nagel at al. eds, Stanford University Press, pp 1-11, 1960.

[BL69] J. R. Büchi and L. H. Landweber. Solving sequential conditions by finitestate strategies. Transactions of the AMS, 138(27):295-311, 1969.

[BS73] J. R. Büchi, D. Siefkes, The Monadic Second-order Theory of all Countable Ordinals, Springer Lecture Notes 328 (1973), pp. 1-126.

[CT02] O. Carton and W.Thomas. The Monadic Theory of Morphic Infinite Words and Generalizations. Inf. Comput. 176(1), pp. 51-65, 2002.

[Ch69] Y. Choueka. Finite Automata on Infinite Structure. Ph.D Thesis, Hebrew University, 1970. 
[ER66] C. Elgot and M. O. Rabin. Decidability and Undecidability of Extensions of Second (First) Order Theory of (Generalized) Successor. J. Symb. Log., 31(2), pp. 169-181, 1966.

[EJ91] E. A. Emerson, C. S. Jutla: Tree Automata, Mu-Calculus and Determinacy (Extended Abstract) FOCS91: 368-377, 1991.

[GTW02] E. Grädel, W. Thomas and T. Wilke. Automata, Logics, and Infinite Games, LNCS 2500, 2002.

[GS83] Y. Gurevich and S. Shelah. Rabin's uniformization problem. The Journal of Symbolic Logic, 48:1105-1119, 1983.

[KV97] O. Kupferman and M.Y. Vardi, Synthesis with incomplete information, In 2nd International Conference on Temporal Logic, pp 91-106, 1997.

[Mc66] R. McNaughton, Testing and generating infinite sequences by a finite automaton, Information and Control 9 (1966), pp. 521-530.

[LS98] S. Lifsches, S. Shelah, Uniformization and skolem functions in the class of trees, Jou. of Symolic Logic, Vol. 63(1) (Mar. 1998), pp. 103-127.

[PP04] D. Perrin and J. E. Pin. Infinite Words Automata, Semigroups, Logic and Games. Pure and Applied Mathematics Vol 141 Elsevier, 2004.

[Rab72] M. O. Rabin. Automata on Infinite Objects and Church's Problem Amer. Math. Soc. Providence, RI, 1972.

[Rab05] A. Rabinovich. On decidability of monadic logic of order over the naturals extended by monadic predicates. 2005 Summer Meeting of the Association for Symbolic Logic, Logic Colloquium 05. The Bulletin of Symbolic Logic 12:343-344, 2006.

[Rab06] A. Rabinovich. The Church problem over $\omega$ expanded by factorial numbers. In preparation, 2006.

[Rab06a] A. Rabinovich. The Church Problem for Countable Ordinals. Submitted, 2006.

[Rab07] A. Rabinovich. On decidability of Monadic logic of order over the naturals extended by monadic predicates. Information and Computation, 2007.

[RT98] A. Rabinovich and B.A. Trakhtenbrot. From Finite Automata toward Hybrid Systems Proceddings of Fundamentals of Computation Theory. Lecture Notes in Computer Science 1450, pp. 411-422, Springer, 1998.

[RS06] A. Rabinovich and A. Shomrat. Selection in the Monadic Theory of Countable Ordinals. Submitted 2006.

[RT06] A. Rabinovich and W. Thomas. Decidable Theories of the Ordering of Natural Numbers with Unary Predicates. In CSL 2006, Springer LNCS 4207, 562-574, 2006.

[RT07] A. Rabinovich and W. Thomas. Logical Refinements of Church's Problem. In CSL 2007, LNCS 4646, 69-83, 2007.

[Rob58] R. M. Robinson. Restricted Set-Theoretical Definitions in Arithmetic. In Proceedings of the AMS Vol. 9, No. 2. pp. 238-242, 1958.

[Sem84] A. Semenov. Logical theories of one-place functions on the set of natural numbers. Mathematics of the USSR - Izvestia, vol. 22, pp 587-618, 1984.

[Se04] O. Serre. Games With Winning Conditions of High Borel Complexity. In ICALP 2004, LNCS volume 3142, pp. 1150-1162, 2004.

[Shel75] S. Shelah. The monadic theory of order. Ann. of Math. 102:379-419, 1975.

[Sie75] D. Siefkes. The recursive sets in certain monadic second order fragments of arithmetic. Arch. Math. Logik, pp71-80, 17(1975).

[Th75] W. Thomas. Das Entscheidungsproblem für einige Erweiterungen der Nachfalger-Arithmetic. Ph. D. Thesis Albert-Ludwigs Universität, 1975.

[Th95] W. Thomas. On the synthesis of strategies in infinite games. In STACS '95, LNCS vo. 900, pp. 1-13. 1995.

[Th03] W. Thomas. Constructing infinite graphs with a decidable MSO-theory. In MFCS03, LNCS 2747, 2003.

[Trak61] B. A. Trakhtenbrot. Finite automata and the logic of one-place predicates. (Russian version 1961). In AMS Transl. 59, 1966, pp. 23-55.

[Wal02] I. Walukiewicz. Monadic second order logic on tree-like structures.TCS 1:275, pp 311-346, 2002. 